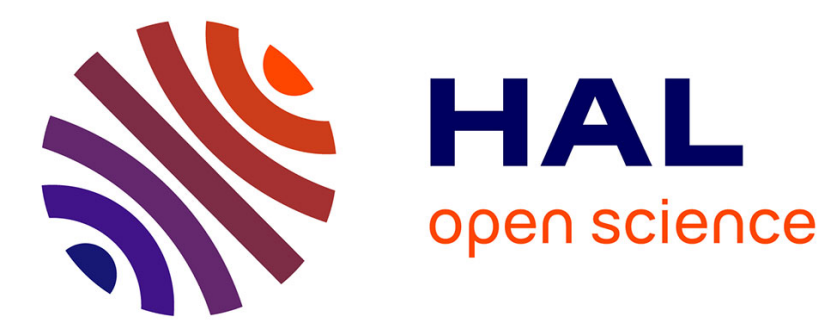

\title{
Proportional Integral regulation control of a one-dimensional semilinear wave equation
}

Hugo Lhachemi, Christophe Prieur, Emmanuel Trélat

\section{To cite this version:}

Hugo Lhachemi, Christophe Prieur, Emmanuel Trélat. Proportional Integral regulation control of a one-dimensional semilinear wave equation. SIAM Journal on Control and Optimization, 2022, 60 (1), pp.1-21. 10.1137/20m1346857 . hal-02872341

\section{HAL Id: hal-02872341 \\ https://hal.science/hal-02872341}

Submitted on 18 Jun 2020

HAL is a multi-disciplinary open access archive for the deposit and dissemination of scientific research documents, whether they are published or not. The documents may come from teaching and research institutions in France or abroad, or from public or private research centers.
L'archive ouverte pluridisciplinaire HAL, est destinée au dépôt et à la diffusion de documents scientifiques de niveau recherche, publiés ou non, émanant des établissements d'enseignement et de recherche français ou étrangers, des laboratoires publics ou privés. 


\title{
PI regulation control of a 1-D semilinear wave equation
}

\author{
Hugo Lhachemi* ${ }^{*}$ Christophe Prieur ${ }^{\dagger} \quad$ Emmanuel Trélat ${ }^{\ddagger}$
}

June 18, 2020

This paper is concerned with the Proportional Integral (PI) regulation control of the left Neumann trace of a one-dimensional semilinear wave equation. The control input is selected as the right Neumann trace. The control design goes as follows. First, a preliminary (classical) velocity feedback is applied in order to shift all but a finite number of the eivenvalues of the underlying unbounded operator into the open left half-plane. We then leverage on the projection of the system trajectories into an adequate Riesz basis to obtain a truncated model of the system capturing the remaining unstable modes. Local stability of the resulting closed-loop infinite-dimensional system composed of the semilinear wave equation, the preliminary velocity feedback, and the PI controller, is obtained through the study of an adequate Lyapunov function. Finally, an estimate assessing the set point tracking performance of the left Neumann trace is derived.

\section{Introduction}

Due to its widespread adoption by industry [8,9], the stabilization and regulation control of finite-dimensional systems by means of Proportional-Integral (PI) controllers has been intensively studied. For this reason, the opportunity of extending PI control strategies to infinite-dimensional systems, and in particular to systems modeled by partial differential equations (PDEs), has attracted much attention in the recent years. Efforts in this research direction were originally devoted to the case of bounded control operators [35, 36] and then extended to unbounded control operators [47]. The study of PI control design combined with high-gain conditions was reported in [32]. More recently, the problem of PI boundary control of linear hyperbolic systems has been reported in a number of works $[12,19,27,48]$. This research direction has then been extended to the case of nonlinear transport equations [11, 14, 20, 23, 37, 45]. The case of the boundary regulation control of the Neumann trace for a linear reaction-diffusion in the presence of an input delay was considered in [29]. The case of the boundary regulation control of the boundary velocity for linear damped wave equations, in the presence of a nonlinearity in the boundary conditions, has been considered in $[10,43]$. A general procedure allowing the addition of an integral component for regulation control to open-loop exponentially stable semigroups with unbounded control operators has been proposed in [41, 42].

This paper is concerned with the PI regulation control of the left Neumann trace of a onedimensional semilinear (undamped) wave equation. The selected control input takes the form of the right Neumann trace. The control design procedure goes as follows. First, inspired by [16], a

\footnotetext{
*University College Dublin, Dublin, Ireland (hugo.lhachemi@ucd.ie).

†Université Grenoble Alpes, CNRS, Grenoble-INP, GIPSA-lab, F-38000, Grenoble (christophe.prieur@gipsalab.fr).

${ }_{\ddagger}^{\ddagger}$ Sorbonne Université, CNRS, Universit?e de Paris, Inria, Laboratoire Jacques-Louis Lions (LJLL), F-75005 Paris, France (emmanuel.trelat@sorbonne-universite.fr).
} 
preliminary (classical) velocity-feedback is applied in order to shift all but a finite number of the eigenvalues of the underlying unbounded operator into the open left half-plan. Then, inspired by the early work [38] later extended in $[15,16,39]$ to semilinear heat and wave PDEs, we leverage on the projection of the system trajectories into a Riesz basis formed by the generalized eigenstructures of the unbounded operator in order to obtain a truncated model capturing the remaining unstable modes. Finally, similarly to [29], this finite dimensional model is augmented to include the integral component of the PI controller, allowing to compute a stabilizing feedback. The local stability of the resulting closed-loop infinite-dimensional system, and the subsequent set point regulation performance, is assessed by a Lyapunov-based argument. The theoretical results are illustrated based on the simulation of an open-loop unstable semilinear wave equation.

The paper is organized as follows. The investigated control problem is introduced in Section 2. The proposed control design procedure is presented in Section 3 in a comprehensive manner. The subsequent stability analysis is carried out in Section 4 while the theoretical results are numerically illustrated in Section 5. Finally, concluding remarks are formulated in Section 6.

\section{Problem setting}

Let $L>0$ and let $f: \mathbb{R} \rightarrow \mathbb{R}$ be a function of class $\mathcal{C}^{2}$. We consider the following wave equation on $(0, L)$ :

$$
\begin{aligned}
& \frac{\partial^{2} y}{\partial t^{2}}=\frac{\partial^{2} y}{\partial x^{2}}+f(y), \\
& y(t, 0)=0, \quad \frac{\partial y}{\partial x}(t, L)=u(t), \\
& y(0, x)=y_{0}(x), \quad \frac{\partial y}{\partial t}(0, x)=y_{1}(x),
\end{aligned}
$$

for $t>0$ and $x \in(0, L)$, where the state is $y(t, \cdot):[0, L] \rightarrow \mathbb{R}$ and the control input is $u(t)$ and applies to the right Neumann trace. The control objective is to design a PI controller in order to locally stabilize the closed-loop system and locally regulate the system output selected as the left Neumann trace:

$$
z(t)=\frac{\partial y}{\partial x}(t, 0)
$$

Definition 1. A function $y_{e} \in \mathcal{C}^{2}([0, L])$ is a steady-state of (1) with associated constant control input $u_{e} \in \mathbb{R}$ and constant system output $z_{e} \in \mathbb{R}$ if

$$
\begin{aligned}
& \frac{\mathrm{d}^{2} y_{e}}{\mathrm{~d} x^{2}}(x)+f\left(y_{e}(x)\right)=0, \quad x \in(0, L), \\
& y_{e}(0)=0, \quad \frac{\mathrm{d} y_{e}}{\mathrm{~d} x}(L)=u_{e}, \\
& z_{e}=\frac{\mathrm{d} y_{e}}{\mathrm{~d} x}(0) .
\end{aligned}
$$

Remark 1. Introducing $F(y)=\int_{0}^{y} f(s) \mathrm{d} s$ for any $y \in \mathbb{R}$, assume that one of the two following properties holds:

- $F(y) \rightarrow+\infty$ when $|y| \rightarrow+\infty$;

- for any $a>0$, when it makes sense, the integral $\int \frac{\mathrm{d} y}{\sqrt{a-F(y)}}$ diverges at $-\infty$ and $+\infty$. 
Then we have the existence of a steady state $y_{e} \in \mathcal{C}^{2}([0, L])$ of $(1)$ associated with any given value of the system output $z_{e} \in \mathbb{R}$. Indeed, define $y \in \mathcal{C}^{2}([0, l))$ with $0<l \leqslant+\infty$ as the maximal solution of $y^{\prime \prime}+f(y)=0$ with $y(0)=0$ and $y^{\prime}(0)=z_{e}$. We only need to assess that $l>L$. Multiplying by $y^{\prime}$ both sides of the ODE satisfied by $y$ and then integrating over $[0, x]$, we observe that $y$ satisfies the conservation law $y^{\prime}(x)^{2}+2 F(y(x))=z_{e}^{2}$ for all $x \in[0, l)$. Hence any of the two above assumptions implies that $y$ and $y^{\prime}$ are bounded on $[0, l)$. Thus $l=+\infty$ and the associated steady state control input is given by $u_{e}=y^{\prime}(L)$.

Given a desired value of the system output $z_{e} \in \mathbb{R}$ and an associated steady state function $y_{e} \in \mathcal{C}^{2}([0, L])$, the control design objective tackled in this paper is to guarantee the local stability of the system (1), when augmented with an adequate control strategy, as well as ensuring the regulation performance, i.e., $z(t)=\frac{\partial y}{\partial x} \rightarrow z_{e}$ when $t \rightarrow+\infty$. To achieve this objective, we introduce the following deviations: $y_{\delta}(t, x)=y(t, x)-y_{e}(x)$ and $u_{\delta}(t)=u(t)-u_{e}$. A Taylor expansion with integral remainder shows that (1) can equivalently be rewritten under the form:

$$
\begin{aligned}
& \frac{\partial^{2} y_{\delta}}{\partial t^{2}}=\frac{\partial^{2} y_{\delta}}{\partial x^{2}}+f^{\prime}\left(y_{e}\right) y_{\delta}+y_{\delta}^{2} \int_{0}^{1}(1-s) f^{\prime \prime}\left(y_{e}+s y_{\delta}\right) \mathrm{d} s, \\
& y_{\delta}(t, 0)=0, \quad \frac{\partial y_{\delta}}{\partial x}(t, L)=u_{\delta}(t), \\
& y_{\delta}(0, x)=y_{0}(x)-y_{e}(x), \quad \frac{\partial y_{\delta}}{\partial t}(0, x)=y_{1}(x),
\end{aligned}
$$

for $t>0$ and $x \in(0, L)$, while the output to be regulated is now expressed as

$$
z_{\delta}(t)=\frac{\partial y_{\delta}}{\partial x}(t, 0)=z(t)-z_{e} .
$$

Finally, following classical proportional integral control design schemes, we introduce the following integral component on the tracking error:

$$
\dot{\zeta}(t)=\frac{\partial y_{\delta}}{\partial x}(t, 0)-z_{r}(t)=z(t)-\left(z_{e}+z_{r}(t)\right)
$$

where $z_{r}(t) \in \mathbb{R}$ is the reference input signal.

Remark 2. It was shown in [29] for a linear reaction-diffusion equation with Dirichlet boundary control that a simple proportional integral controller can be used to successfully control a Neumann trace. The control design was performed on a finite-dimensional truncated model capturing the unstable modes of the infinite dimensional system while assessing the stability of the full infinitedimensional system via a Lyapunov-based argument. Such an approach cannot be directly applied to the case of the wave equation studied in this paper due to the fact that, even in the case of a linear function $f$, the open-loop system might exhibit an infinite number of unstable modes. To avoid this pitfall, we borrow the following remark from [16]. In the case $f=0$, the control input $u_{\delta}(t)=-\alpha \frac{\partial y_{\delta}}{\partial t}(t, L)$, with $\alpha>0$, ensures the exponential decay of the energy function defined as:

$$
E(t)=\int_{0}^{L}\left(\frac{\partial y_{\delta}}{\partial t}(t, x)\right)^{2}+\left(\frac{\partial y_{\delta}}{\partial x}(t, x)\right)^{2} \mathrm{~d} x .
$$

Thus, as suggested in [16], a suitable control input candidate for (3) takes the form:

$$
u_{\delta}(t)=-\alpha \frac{\partial y_{\delta}}{\partial t}(t, L)+v(t)
$$


where $\alpha>0$ is to be selected and $v(t)$ is an auxiliary command input. In particular, it was shown in [16] that, in the presence of the nonlinear term $f$, the velocity feedback can be used to locally stabilize all but possibly a finite number of the modes of the system. Then the authors showed that the design of the auxiliary control input $v$ can be performed by pole shifting on a finite dimensional truncated model to achieve the stabilization of the remaining unstable modes. The stability of the resulting closed-loop system was assessed via the introduction of a suitable Lyapunov function. In this paper, we propose to take advantage of such a control design strategy in order to achieve the regulation of the following Neumann trace by means of a proportional integral control design scheme via the introduced integral component (5).

\section{Control design}

\subsection{Equivalent homogeneous problem}

By introducing the change of variable:

$$
w^{1}(t, x)=y_{\delta}(t, x), \quad w^{2}(t, x)=\frac{\partial y_{\delta}}{\partial t}(t, x)-\frac{x}{\alpha L} v(t),
$$

we obtain from the wave equation (3), the integral component (5), and the control strategy (6) that

$$
\begin{aligned}
& \frac{\partial w^{1}}{\partial t}=w^{2}+\frac{x}{\alpha L} v(t), \\
& \frac{\partial w^{2}}{\partial t}=\frac{\partial^{2} w^{1}}{\partial x^{2}}+f^{\prime}\left(y_{e}\right) w^{1}+r(t, x)-\frac{x}{\alpha L} \dot{v}(t), \\
& \dot{\zeta}(t)=\frac{\partial w^{1}}{\partial x}(t, 0)-z_{r}(t), \\
& w^{1}(t, 0)=0, \quad \frac{\partial w^{1}}{\partial x}(t, L)+\alpha w^{2}(t, L)=0, \\
& w^{1}(0, x)=y_{0}(x)-y_{e}(x), \quad w^{2}(0, x)=y_{1}(x)-\frac{x}{\alpha L} v(0), \\
& \zeta(0)=\zeta_{0}
\end{aligned}
$$

for $t>0$ and $x \in(0, L)$, with the residual term

$$
r(t, x)=\left(w^{1}(t, x)\right)^{2} \int_{0}^{1}(1-s) f^{\prime \prime}\left(y_{e}(x)+s w^{1}(t, x)\right) \mathrm{d} s .
$$

Remark 3. A more classical change of variable for (3) with control input $u$ given by (6) is generally obtained by setting

$$
w(t, x)=y_{\delta}(t, x)-\frac{x(x-L)}{L} v(t) .
$$

In that case, (3) with $u$ given by (6) yields

$$
\begin{aligned}
& \frac{\partial^{2} w}{\partial t^{2}}=\frac{\partial^{2} w}{\partial x^{2}}+f^{\prime}\left(y_{e}\right) w-\frac{x(x-L)}{L} \ddot{v}(t)+\left(\frac{x(x-L)}{L} f^{\prime}\left(y_{e}\right)+\frac{2}{L}\right) v(t)+r(t, x), \\
& w(t, 0)=0, \quad \frac{\partial w}{\partial x}(t, L)+\alpha \frac{\partial w}{\partial t}(t, L)=0, \\
& w(0, x)=y_{0}(x)-y_{e}(x)-\frac{x(x-L)}{L} v(0), \quad \frac{\partial w}{\partial t}(0, x)=y_{1}(x)-\frac{x(x-L)}{L} \dot{v}(0)
\end{aligned}
$$


with

$$
\begin{aligned}
& r(t, x)= \\
& \left(w(t, x)+\frac{x(x-L)}{L} v(t)\right)^{2} \int_{0}^{1}(1-s) f^{\prime \prime}\left(y_{e}(x)+s\left(w(t, x)+\frac{x(x-L)}{L} v(t)\right)\right) \mathrm{d} s .
\end{aligned}
$$

However, this change of variable (10) induces the occurrence of a $\ddot{v}$ term in (11a), while only a $\dot{v}$ term appears in (8a-8b). Thus, in the subsequent procedure, the consideration of the change of variable (7) instead of (10) will allow a reduction of the complexity of the controller architecture by avoiding the introduction of an extra additional integral component.

We now introduce the Hilbert space

$$
\mathcal{H}=\left\{\left(w^{1}, w^{2}\right) \in H^{1}(0, L) \times L^{2}(0, L): w^{1}(0)=0\right\}
$$

endowed with the inner product

$$
\left\langle\left(w^{1}, w^{2}\right),\left(z^{1}, z^{2}\right)\right\rangle=\int_{0}^{L}\left(w^{1}\right)^{\prime}(x) \overline{\left(z^{1}\right)^{\prime}(x)}+w^{2}(x) \overline{z^{2}(x)} \mathrm{d} x .
$$

Defining the following state vector

$$
W(t)=\left(w^{1}(t, \cdot), w^{2}(t, \cdot)\right) \in \mathcal{H},
$$

the wave equation with integral component (8) can be rewritten under the abstract form

$$
\begin{aligned}
& \frac{\mathrm{d} W}{\mathrm{~d} t}(t)=\mathcal{A} W(t)+a v(t)+b \dot{v}(t)+R(t, \cdot), \\
& \dot{\zeta}(t)=\frac{\partial w^{1}}{\partial x}(t, 0)-z_{r}(t), \\
& W(0, x)=\left(y_{0}(x)-y_{e}(x), y_{1}(x)-\frac{x}{\alpha L} v(0)\right), \\
& \zeta(0)=\zeta_{0} .
\end{aligned}
$$

for $t>0$ and $x \in(0, L)$, where

$$
\mathcal{A}=\left(\begin{array}{cc}
0 & \mathrm{Id} \\
\mathcal{A}_{0} & 0
\end{array}\right)
$$

with $\mathcal{A}_{0}=\Delta+f^{\prime}\left(y_{e}\right)$ Id on the domain

$$
\begin{array}{r}
D(\mathcal{A})=\left\{\left(w^{1}, w^{2}\right) \in \mathcal{H}: w^{1} \in H^{2}(0, L), w^{2} \in H^{1}(0, L),\right. \\
\left.w^{2}(0)=0,\left(w^{1}\right)^{\prime}(L)+\alpha w^{2}(L)=0\right\},
\end{array}
$$

and $a, b, R(t, \cdot) \in \mathcal{H}$ are defined by

$$
a(x)=(x /(\alpha L), 0), \quad b(x)=(0,-x /(\alpha L)), \quad R(t, x)=(0, r(t, x)) .
$$

We have $R(t, \cdot) \in \mathcal{H}$ because $r(t, \cdot) \in L^{2}(0, L)$, which is a direct consequence of the facts that $w^{1}(t, \cdot) \in H^{1}(0, L) \subset L^{\infty}(0, L), f^{\prime \prime}$ is continuous on $\mathbb{R}$, and $y_{e}$ is continuous on $[0, L]$.

Remark 4. It is well-known that the operator $\mathcal{A}$ generates a $C_{0}$-semigroup [46]. Moreover, because the Neumann trace is $\mathcal{A}$-admissible, the application of [47, Lemma 1] shows that the augmentation of $\mathcal{A}$ with the integral component $\zeta$ still generates a $C_{0}$-semigroup. As $\dot{v}$ is seen as the control 
input, the state-space vector can further be augmented to include $v$, and the associated augmented operator also generates a $C_{0}$-semigroup. Now, noting that the residual term (9) can be rewritten under the form

$$
r(t, x)=\int_{y_{e}(x)}^{w^{1}(t, x)+y_{e}(x)}\left(w^{1}(t, x)+y_{e}(x)-s\right) f^{\prime \prime}(s) \mathrm{d} s,
$$

one can see that $w^{1} \rightarrow \int_{y_{e}}^{w^{1}+y_{e}}\left(w^{1}+y_{e}-s\right) f^{\prime \prime}(s) \mathrm{d} s$, when seen as a function from $\left\{w^{1} \in H^{1}(0, L)\right.$ : $\left.w^{1}(0)=0\right\}$ to $L^{2}(0, L)$, is continuously differentiable. Consequently, the well-posedness of (12) follows from classical results [34]. In the subsequent developments, we will consider for initial conditions $W(0) \in D(\mathcal{A})$, continuously differentiable reference inputs $z_{r}$, and a control input $\dot{v}$ that will take the form of a state-feedback, the concept of classical solution for (12) on its maximal interval of definition $\left[0, T_{\max }\right)$ with $0<T_{\max } \leqslant+\infty$, i.e. $W \in \mathcal{C}^{0}\left(\left[0, T_{\max }\right) ; D(\mathcal{A})\right) \cap$ $\mathcal{C}^{1}\left(\left[0, T_{\max }\right) ; \mathcal{H}\right)$.

\subsection{Properties of the operator $\mathcal{A}$}

First, we explicit in the following lemma the adjoint operator $\mathcal{A}^{*}$.

Lemma 1. The adjoint operator of $\mathcal{A}$ is defined on

$$
\begin{array}{r}
D\left(\mathcal{A}^{*}\right)=\left\{\left(z^{1}, z^{2}\right) \in \mathcal{H}: z^{1} \in H^{2}(0, L), z^{2} \in H^{1}(0, L),\right. \\
\left.z^{2}(0)=0,\left(z^{1}\right)^{\prime}(L)-\alpha z^{2}(L)=0\right\}
\end{array}
$$

by

$$
\mathcal{A}^{*}\left(z^{1}, z^{2}\right)=\left(-z^{2}-g,-\left(z^{1}\right)^{\prime \prime}\right)
$$

where $g \in \mathcal{C}^{2}([0, L])$ is uniquely defined by

$$
\begin{aligned}
& g^{\prime \prime}=f^{\prime}\left(y_{e}\right) z^{2}, \\
& g(0)=g^{\prime}(L)=0 .
\end{aligned}
$$

Proof. We write $\mathcal{A}=\mathcal{A}_{t r}+\mathcal{A}_{p}$ with

$$
\mathcal{A}_{t r}=\left(\begin{array}{cc}
0 & \mathrm{Id} \\
\Delta & 0
\end{array}\right), \quad \mathcal{A}_{p}=\left(\begin{array}{cc}
0 & 0 \\
f^{\prime}\left(y_{e}\right) \text { Id } & 0
\end{array}\right)
$$

where $\mathcal{A}_{t r}$ is an unbounded operator defined on the same domain as $\mathcal{A}$ while $\mathcal{A}_{p}$ in defined on $\mathcal{H}$. As $\mathcal{A}_{p}$ is bounded, straightforward computations show that $\mathcal{A}_{p}^{*}=(-g, 0)$ where $g$ is defined as in the statement of the Lemma. It remains to compute $\mathcal{A}_{t r}^{*}$. To do so, one can observe that $0 \in \rho\left(\mathcal{A}_{t r}\right)$ with

$$
\mathcal{A}_{t r}^{-1} w=\left(-\alpha w^{1}(L) x+\int_{0}^{x} \int_{L}^{\xi} w^{2}(s) \mathrm{d} s \mathrm{~d} \xi, w^{1}\right), \quad \forall w=\left(w^{1}, w^{2}\right) \in \mathcal{H} .
$$

Since $\mathcal{A}_{t r}^{-1}$ is bounded, straightforward computations show that

$$
\left(\mathcal{A}_{t r}^{-1}\right)^{*} w=\left(-\alpha w^{1}(L) x-\int_{0}^{x} \int_{L}^{\xi} w^{2}(s) \mathrm{d} s \mathrm{~d} \xi,-w^{1}\right), \quad \forall w=\left(w^{1}, w^{2}\right) \in \mathcal{H} .
$$

We deduce the claimed result by computing the inverse of the latter operator.

The strategy reported in this paper relies on the concept of Riesz bases. This concept is recalled in the following definition. 
Definition 2. A family of vectors $\left(e_{k}\right)_{k \in \mathbb{Z}}$ of $\mathcal{H}$ is a Riesz basis if this family is maximal and there exist constants $m_{r}, M_{R}>0$ such that for any $N \geqslant 0$ and any $c_{-n_{0}}, \ldots, c_{n_{0}} \in \mathbb{C}$,

$$
m_{R} \sum_{|k| \leqslant N}\left|c_{k}\right|^{2} \leqslant\left\|\sum_{|k| \leqslant N} c_{k} e_{k}\right\|_{\mathcal{H}}^{2} \leqslant M_{R} \sum_{|k| \leqslant N}\left|c_{k}\right|^{2} .
$$

The dual Riesz basis of $\left(e_{k}\right)_{k \in \mathbb{Z}}$ is the unique family of vectors $\left(f_{k}\right)_{k \in \mathbb{Z}}$ of $\mathcal{H}$ which is such that $\left\langle e_{k}, f_{l}\right\rangle_{\mathcal{H}}=\delta_{k, l} \in\{0,1\}$ with $\delta_{k, l}=1$ if and only if $k=l$.

We can now introduce the following properties of the operator $\mathcal{A}$. These properties, expect the last item, are retrieved from [16, Lemmas 2 and 5].

Lemma 2. Let $\alpha>1$. There exists a Riesz basis $\left(e_{k}\right)_{k \in \mathbb{Z}}$ of $\mathcal{H}$ consisting of generalized eigenfunctions of $\mathcal{A}$, associated to the eigenvalues $\left(\lambda_{k}\right)_{k \in \mathbb{Z}}$ and with dual Riesz basis $\left(f_{k}\right)_{k \in \mathbb{Z}}$, such that:

1. $e_{k} \in D(\mathcal{A})$ and $\left\|e_{k}\right\|_{\mathcal{H}}=1$ for every $k \in \mathbb{Z}$;

2. each eigenvalue $\lambda_{k}$ is geometrically simple;

3. there exists $n_{0} \geqslant 0$ such that, for any $k \in \mathbb{Z}$ with $|k| \geqslant n_{0}+1$, the eigenvalue $\lambda_{k}$ is algebraically simple and satisfies

$$
\lambda_{k}=\frac{1}{2 L} \log \left(\frac{\alpha-1}{\alpha+1}\right)+i \frac{k \pi}{L}+O\left(\frac{1}{|k|}\right)
$$

as $|k| \rightarrow+\infty$.

4. if $k \geqslant n_{0}+1$, then $e_{k}$ (resp. $f_{k}$ ) is an eigenfunction of $\mathcal{A}\left(\right.$ resp. $\left.\mathcal{A}^{*}\right)$ associated with the algebraically simple eigenvalue $\lambda_{k}$ (resp. $\overline{\lambda_{k}}$ );

5. for every $k \geqslant n_{0}+1$, one has $e_{k}=\overline{e_{-k}}$ and $f_{k}=\overline{f_{-k}}$;

6. for every $|k| \leqslant n_{0}$, there holds

$$
\begin{array}{r}
\mathcal{A} e_{k} \in \operatorname{span}\left\{e_{p}:|p| \leqslant n_{0}\right\}, \\
\mathcal{A}^{*} f_{k} \in \operatorname{span}\left\{f_{p}:|p| \leqslant n_{0}\right\} ;
\end{array}
$$

7. introducing $e_{k}=\left(e_{k}^{1}, e_{k}^{2}\right)$, one has $\left(e_{k}^{1}\right)^{\prime}(0)=O(1)$ as $|k| \rightarrow+\infty$.

The proof of Lemma A, which is essentially extracted from [16], is placed in annex for selfcompleteness of the manuscript.

In the remainder of the paper, we select the constant $\alpha>1$ such that

$$
\frac{1}{2 L} \log \left(\frac{\alpha-1}{\alpha+1}\right)<-1 \text {. }
$$

Based on the asymptotic behavior (16), only a finite number of eigenvalues might have a non negative real part. Thus, without loss of generality, we also select the integer $n_{0} \geqslant 0$ provided by Lemma 2 large enough such that $\operatorname{Re} \lambda_{k}<-1$ for all $|k| \geqslant n_{0}+1$.

Remark 5. The state-space can be written as $\mathcal{H}=\mathcal{H}_{1} \oplus \mathcal{H}_{2}$ with the subspaces $\mathcal{H}_{1}=\operatorname{span}\left\{e_{p}\right.$ : $\left.|p| \leqslant n_{0}\right\}$ and $\mathcal{H}_{2}=\overline{\operatorname{span}\left\{e_{p}:|p| \geqslant n_{0}+1\right\}}$. Introducing $\pi_{1}, \pi_{2}$ the projectors associated with this decomposition, Lemma 2 shows that $\mathcal{A}$ takes the form $\mathcal{A}=\mathcal{A}_{1} \pi_{1}+\mathcal{A}_{2} \pi_{2}$ where $\mathcal{A}_{1} \in \mathcal{L}\left(\mathcal{H}_{1}\right)$ and 
$\mathcal{A}_{2}: D\left(\mathcal{A}_{2}\right) \subset \mathcal{H}_{2} \rightarrow \mathcal{H}_{2}$ with $D\left(\mathcal{A}_{2}\right)=D(\mathcal{A}) \cap \mathcal{H}_{2}$. Moreover, Lemma 2 shows that $\mathcal{A}_{2}$ is a Riesz spectral operator. Then, as $D(\mathcal{A})=\mathcal{H}_{1} \oplus D\left(\mathcal{A}_{2}\right)$, we obtain that

$$
D(\mathcal{A})=\left\{\sum_{k \in \mathbb{Z}} w_{k} e_{k}: \sum_{k \in \mathbb{Z}}\left|\lambda_{k} w_{k}\right|^{2}<\infty\right\}
$$

and, for any $w \in D(\mathcal{A})$,

$$
\mathcal{A} w=\mathcal{A}_{1} \pi_{1} w+\sum_{|k| \geqslant n_{0}+1} \lambda_{k}\left\langle w, f_{k}\right\rangle e_{k},
$$

where the equality holds in $\mathcal{H}$-norm. Now, for any $w \in D(\mathcal{A})$, consider the series expansion $w=$ $\left(w^{1}, w^{2}\right)=\sum_{k \in \mathbb{Z}}\left\langle w, f_{k}\right\rangle e_{k}$. In particular, one has $w^{1}=\sum_{k \in \mathbb{Z}}\left\langle w, f_{k}\right\rangle e_{k}^{1}$ in $H^{1}$-norm. Moreover, from (18) we have that $\mathcal{A} w=\sum_{k \in \mathbb{Z}}\left\langle w, f_{k}\right\rangle \mathcal{A} e_{k}$ and thus $\mathcal{A}_{0} w^{1}=\sum_{k \in \mathbb{Z}}\left\langle w, f_{k}\right\rangle \mathcal{A}_{0} e_{k}^{1}$ in $L^{2}$-norm. Since $f^{\prime}\left(y_{e}\right) \in L^{\infty}(0, L)$, the expansion of the latter identity shows that $\left(w^{1}\right)^{\prime \prime}=\sum_{k \in \mathbb{Z}}\left\langle w, f_{k}\right\rangle\left(e_{k}^{1}\right)^{\prime \prime}$ in $L^{2}$-norm. Consequently, $w^{1}=\sum_{k \in \mathbb{Z}}\left\langle w, f_{k}\right\rangle e_{k}^{1}$ in $H^{2}$-norm and thus, by the continuous embed$\operatorname{ding} H^{1}(0, L) \subset L^{\infty}(0, L)$,

$$
\left(w^{1}\right)^{\prime}(0)=\sum_{k \in \mathbb{Z}}\left\langle w, f_{k}\right\rangle\left(e_{k}^{1}\right)^{\prime}(0)
$$

The latter series expansion will be intensively used in the remainder of this paper.

\subsection{Spectral reduction and truncated model}

Introducing, for every $k \in \mathbb{Z}$,

$$
w_{k}(t)=\left\langle W(t), f_{k}\right\rangle_{\mathcal{H}}, \quad a_{k}=\left\langle a, f_{k}\right\rangle_{\mathcal{H}}, \quad b_{k}=\left\langle b, f_{k}\right\rangle_{\mathcal{H}}, \quad r_{k}(t)=\left\langle R(t, \cdot), f_{k}\right\rangle_{\mathcal{H}},
$$

we obtain from (12a), that

$$
\begin{aligned}
\dot{w}_{k}(t) & =\left\langle\mathcal{A} W(t), f_{k}\right\rangle_{\mathcal{H}}+a_{k} v(t)+b_{k} \dot{v}(t)+r_{k}(t) \\
& =\left\langle W(t), \mathcal{A}^{*} f_{k}\right\rangle_{\mathcal{H}}+a_{k} v(t)+b_{k} \dot{v}(t)+r_{k}(t)
\end{aligned}
$$

Recalling that $\mathcal{A}^{*} f_{k}=\overline{\lambda_{k}} f_{k}$ for $|k| \geqslant n_{0}+1$, we obtain that

$$
\dot{w}_{k}(t)=\lambda_{k} w_{k}(t)+a_{k} v(t)+b_{k} \dot{v}(t)+r_{k}(t), \quad|k| \geqslant n_{0}+1 .
$$

Moreover, after possibly linear recombination ${ }^{1}$ of $\left(e_{k}\right)_{|k| \leqslant N_{0}}$ and $\left(f_{k}\right)_{|k| \leqslant N_{0}}$, which we still denote by $\left(e_{k}\right)_{|k| \leqslant N_{0}}$ and $\left(f_{k}\right)_{|k| \leqslant N_{0}}$, to obtain matrices with real coefficients, we infer from (17) the existence of a matrix $A_{0} \in \mathbb{R}^{\left(2 n_{0}+1\right) \times\left(2 n_{0}+1\right)}$ such that

$$
\dot{X}_{0}(t)=A_{0} X_{0}(t)+B_{0,1} v(t)+B_{0,2} \dot{v}(t)+R_{0}(t)
$$

where

$$
\begin{gathered}
X_{0}(t)=\left[\begin{array}{c}
w_{-n_{0}}(t) \\
\vdots \\
w_{n_{0}}(t)
\end{array}\right] \in \mathbb{R}^{2 n_{0}+1}, \quad B_{0,1}=\left[\begin{array}{c}
a_{-n_{0}} \\
\vdots \\
a_{n_{0}}
\end{array}\right] \in \mathbb{R}^{2 n_{0}+1}, \\
B_{0.2}=\left[\begin{array}{c}
b_{-n_{0}} \\
\vdots \\
b_{n_{0}}
\end{array}\right] \in \mathbb{R}^{2 n_{0}+1}, \quad R_{0}(t)=\left[\begin{array}{c}
r_{-n_{0}}(t) \\
\vdots \\
r_{n_{0}}(t)
\end{array}\right] \in \mathbb{R}^{2 n_{0}+1} .
\end{gathered}
$$

\footnotetext{
${ }^{1}$ In that case, all the properties stated by Lemma 2 remain true except that $\left(e_{k}\right)_{|k| \leqslant N_{0}}$ might not be generalized eigenvectors of $\mathcal{A}$.
} 
We augment the state-space representation (21) with the actual control input $v$ as follows:

$$
\dot{X}_{1}(t)=A_{1} X_{1}(t)+B_{1} v_{d}(t)+R_{1}(t)
$$

where

$$
\begin{aligned}
X_{1}(t) & =\left[\begin{array}{c}
v(t) \\
X_{0}(t)
\end{array}\right] \in \mathbb{R}^{2 n_{0}+2}, \quad v_{d}(t)=\dot{v}(t), \quad R_{1}(t)=\left[\begin{array}{c}
0 \\
R_{0}(t)
\end{array}\right] \in \mathbb{R}^{2 n_{0}+2} \\
A_{1} & =\left[\begin{array}{cc}
0 & 0 \\
B_{0,1} & A_{0}
\end{array}\right] \in \mathbb{R}^{\left(2 n_{0}+2\right) \times\left(2 n_{0}+2\right)}, \quad B_{1}=\left[\begin{array}{c}
1 \\
B_{0,2}
\end{array}\right] \in \mathbb{R}^{2 n_{0}+2} .
\end{aligned}
$$

We now further augment the latter state-space representation to include the integral component of the PI controller. First, we note from (19) that the dynamics of the integral component $\zeta$ satisfies

$$
\dot{\zeta}(t)=\sum_{k \in \mathbb{Z}} w_{k}(t)\left(e_{k}^{1}\right)^{\prime}(0)-z_{r}(t) .
$$

We observe that the $\zeta$-dynamics involves all the coefficients of projection $w_{k}(t)$, with $k \in \mathbb{Z}$, hence cannot be used to augment (22). This motivates the introduction of the following change of variable:

$$
\begin{aligned}
\xi(t) & =\zeta(t)-\sum_{|k| \geqslant n_{0}+1} \frac{\left(e_{k}^{1}\right)^{\prime}(0)}{\lambda_{k}} w_{k}(t) \\
& =\zeta(t)-2 \sum_{k \geqslant n_{0}+1} \operatorname{Re}\left\{\frac{\left(e_{k}^{1}\right)^{\prime}(0)}{\lambda_{k}} w_{k}(t)\right\}
\end{aligned}
$$

where, using Cauchy-Schwarz inequality, the series is convergent because $\left|\lambda_{k}\right| \sim \frac{|k| \pi}{l}$ and $\left(e_{k}^{1}\right)^{\prime}(0)=$ $O(1)$ as $|k| \rightarrow+\infty$. Moreover, the time derivative of $\xi$ is given by

$$
\begin{aligned}
\dot{\xi}(t) & =\dot{\zeta}(t)-\sum_{|k| \geqslant n_{0}+1} \frac{\left(e_{k}^{1}\right)^{\prime}(0)}{\lambda_{k}} \dot{w}_{k}(t) \\
& =\sum_{|k| \leqslant n_{0}} w_{k}(t)\left(e_{k}^{1}\right)^{\prime}(0)+\alpha_{0} v(t)+\beta_{0} \dot{v}(t)-\gamma(t) \\
& =L_{1} X_{1}(t)+\beta_{0} v_{d}(t)-\gamma(t),
\end{aligned}
$$

where

$$
\begin{aligned}
\alpha_{0} & =-\sum_{|k| \geqslant n_{0}+1} \frac{\left(e_{k}^{1}\right)^{\prime}(0)}{\lambda_{k}} a_{k}=-2 \sum_{k \geqslant n_{0}+1} \operatorname{Re}\left\{\frac{\left(e_{k}^{1}\right)^{\prime}(0)}{\lambda_{k}} a_{k}\right\}, \\
\beta_{0} & =-\sum_{|k| \geqslant n_{0}+1} \frac{\left(e_{k}^{1}\right)^{\prime}(0)}{\lambda_{k}} b_{k}=-2 \sum_{k \geqslant n_{0}+1} \operatorname{Re}\left\{\frac{\left(e_{k}^{1}\right)^{\prime}(0)}{\lambda_{k}} b_{k}\right\}, \\
\gamma(t) & =z_{r}(t)+\sum_{|k| \geqslant n_{0}+1} \frac{\left(e_{k}^{1}\right)^{\prime}(0)}{\lambda_{k}} r_{k}(t)=z_{r}(t)+2 \sum_{k \geqslant n_{0}+1} \operatorname{Re}\left\{\frac{\left(e_{k}^{1}\right)^{\prime}(0)}{\lambda_{k}} r_{k}(t)\right\},
\end{aligned}
$$

and

$$
L_{1}=\left[\begin{array}{llll}
\alpha_{0} & \left(e_{-n_{0}}^{1}\right)^{\prime}(0) & \ldots & \left(e_{n_{0}}^{1}\right)^{\prime}(0)
\end{array}\right] \in \mathbb{R}^{1 \times\left(2 n_{0}+2\right)} .
$$

Thus, with the introduction of

$$
X(t)=\left[\begin{array}{c}
X_{1}(t) \\
\xi(t)
\end{array}\right] \in \mathbb{R}^{2 n_{0}+3}, \quad A=\left[\begin{array}{cc}
A_{1} & 0 \\
L_{1} & 0
\end{array}\right] \in \mathbb{R}^{\left(2 n_{0}+3\right) \times\left(2 n_{0}+3\right)},
$$




$$
B=\left[\begin{array}{c}
B_{1} \\
\beta_{0}
\end{array}\right] \in \mathbb{R}^{2 n_{0}+3}, \quad \Gamma(t)=\left[\begin{array}{c}
R_{1}(t) \\
-\gamma(t)
\end{array}\right] \in \mathbb{R}^{2 n_{0}+3},
$$

we obtain the truncated model

$$
\dot{X}(t)=A X(t)+B v_{d}(t)+\Gamma(t) .
$$

Putting (20) and (27) together, we obtain that the wave equation with integral component (12) admits the following equivalent representation used for both control design and stability analysis:

$$
\begin{aligned}
& \dot{X}(t)=A X(t)+B v_{d}(t)+\Gamma(t), \\
& \dot{w}_{k}(t)=\lambda_{k} w_{k}(t)+a_{k} v(t)+b_{k} v_{d}(t)+r_{k}(t), \quad|k| \geqslant n_{0}+1 .
\end{aligned}
$$

Remark 6. The representation (28) shows that the dynamics of the wave equation with integral component (12) can be split into two parts. The first part, given by (28a), consists of an ODE capturing the unstable dynamics plus a certain number of slow stable modes of the system. The second part, given by (28b) and referred to as the residual dynamics, captures the stable dynamics of the system which are such that $\operatorname{Re} \lambda_{k}<-1$. The control strategy consists now into the two following steps. First, a state-feedback is designed to locally stabilize (28a). Then, a stability analysis is carried out to assess that such a control strategy achieves both the local stabilization of (28), as well as the fulfillment of the output regulation of the Neumann trace (4).

\subsection{Control strategy and closed-loop dynamics}

The control design strategy consists in the design of a stabilizing state-feedback for (28a). Such a pole shifting is allowed by the following result.

Lemma 3. The pair $(A, B)$ satisfies the Kalman condition.

Proof. From (26), the Hautus test easily shows that $(A, B)$ satisfies the Kalman condition if and only if $\left(A_{1}, B_{1}\right)$ satisfies the Kalman condition and the square matrix

$$
T=\left[\begin{array}{ll}
A_{1} & B_{1} \\
L_{1} & \beta_{0}
\end{array}\right] \in \mathbb{R}^{\left(2 n_{0}+3\right) \times\left(2 n_{0}+3\right)}
$$

is invertible.

We first show the following preliminary result: for any $\lambda \in \mathbb{C}$ and $z \in D\left(\mathcal{A}^{*}\right),\langle a+\lambda b, z\rangle_{\mathcal{H}}=0$ and $\mathcal{A}^{*} z=\bar{\lambda} z$ implies $z=0$. Recall based on Lemma 1 that $\mathcal{A}^{*} z=\bar{\lambda} z$ gives

$$
\begin{aligned}
& z^{2}+g=-\bar{\lambda} z^{1} \\
& \left(z^{1}\right)^{\prime \prime}=-\bar{\lambda} z^{2} \\
& g^{\prime \prime}=f^{\prime}\left(y_{e}\right) z^{2} \\
& z^{1}(0)=z^{2}(0)=g(0)=g^{\prime}(L)=0, \\
& \left(z^{1}\right)^{\prime}(L)-\alpha z^{2}(L)=0 .
\end{aligned}
$$

From the definition (14) of $a, b \in \mathcal{H}$ one has

$$
\begin{aligned}
\langle a+\lambda b, z\rangle_{\mathcal{H}} & =\int_{0}^{L}\left(\frac{x}{\alpha L}\right)^{\prime} \overline{\left(z^{1}\right)^{\prime}(x)}-\lambda \frac{x}{\alpha L} \overline{z^{2}(x)} \mathrm{dx} \\
& =\left[\frac{x}{\alpha L} \overline{\left(z^{1}\right)^{\prime}(x)}\right]_{x=0}^{x=L}-\int_{0}^{L} \frac{x}{\alpha L} \overline{\left(\left(z^{1}\right)^{\prime \prime}(x)+\bar{\lambda} z^{2}(x)\right)} \mathrm{dx}
\end{aligned}
$$




$$
=\frac{1}{\alpha} \overline{\left(z^{1}\right)^{\prime}(L)}
$$

where we have used (29b). Thus we have $\left(z^{1}\right)^{\prime}(L)=0$. Then (29e) shows that $z^{2}(L)=0$ and we infer from (29a) and (29d) that $\left(z^{2}\right)^{\prime}(L)=0$. Moreover, taking twice the derivative of (29a) and using (29b-29c), we obtain that $\left(z^{2}\right)^{\prime \prime}+\left(f^{\prime}\left(y_{e}\right)-(\bar{\lambda})^{2}\right) z^{2}=0$. By Cauchy uniqueness, we deduce that $z^{2}=0$. Using $(29 \mathrm{~b}),(29 \mathrm{~d})$ and $\left(z^{1}\right)^{\prime}(L)=0$, we reach the conclusion $z=0$.

Assume now that $\left(A_{1}, B_{1}\right)$ does not satisfy the Kalman condition. From (23b), the Hautus test shows the existence of $\lambda \in \mathbb{C}, x_{1} \in \mathbb{C}$, and $x_{2} \in \mathbb{C}^{2 n_{0}+1}$, with either $x_{1} \neq 0$ or $x_{2} \neq 0$, such that

$$
\begin{aligned}
& x_{2}^{*} B_{0,1}=\lambda x_{1}^{*}, \\
& x_{2}^{*} A_{0}=\lambda x_{2}^{*}, \\
& x_{1}^{*}+x_{2}^{*} B_{0,2}=0 .
\end{aligned}
$$

This implies the existence of $x_{2} \neq 0$ such that

$$
\begin{aligned}
& A_{0}^{*} x_{2}=\bar{\lambda} x_{2}, \\
& x_{2}^{*}\left(B_{0,1}+\lambda B_{0,2}\right)=0
\end{aligned}
$$

where $B_{0,1}+\lambda B_{0,2}=\left(\left\langle a+\lambda b, f_{k}\right\rangle_{\mathcal{H}}\right)_{-n_{0} \leqslant k \leqslant n_{0}}$. Noting that $A_{0}^{*}$ is the matrix of $\mathcal{A}^{*}$ in $\left(f_{k}\right)_{|k| \leqslant n_{0}}$, this shows the existence of a nonzero vector $z \in D\left(\mathcal{A}^{*}\right)$ such that $\langle a+\lambda b, z\rangle_{\mathcal{H}}=0$ and $\mathcal{A}^{*} z=\bar{\lambda} z$. The result of the previous paragraph leads to the contraction $z=0$. Hence $\left(A_{1}, B_{1}\right)$ does satisfy the Kalman condition.

It remains to show that the matrix $T$ is invertible. Let a vector

$$
X_{e}=\left[\begin{array}{lllll}
v_{e} & w_{-n_{0}, e} & \cdots & w_{-n_{0}, e} & v_{d, e}
\end{array}\right]^{\top} \in \mathbb{R}^{2 n_{0}+3}
$$

be an element of the kernel of $T$. Then, by expanding $T X_{e}=0$, we obtain that

$$
\begin{aligned}
0 & =v_{d, e}, \\
0 & =A_{0}\left[\begin{array}{c}
w_{-n_{0}, e} \\
\vdots \\
w_{-n_{0}, e}
\end{array}\right]+B_{0,1} v_{e}, \\
0 & =\sum_{|k| \leqslant n_{0}} w_{k, e}\left(e_{k}^{1}\right)^{\prime}(0)-\left(\sum_{|k| \geqslant n_{0}+1} \frac{\left(e_{k}^{1}\right)^{\prime}(0)}{\lambda_{k}} a_{k}\right) v_{e} .
\end{aligned}
$$

We define, for $|k| \geqslant n_{0}+1, w_{k, e}=-\frac{a_{k}}{\lambda_{k}} v_{e}$. Then, as $\left(w_{k, e}\right)_{k \in \mathbb{Z}}$ is square summable, we can introduce $w_{e}=\sum_{k \in \mathbb{Z}} w_{k, e} e_{k} \in \mathcal{H}$. We obtain that

$$
\begin{aligned}
0 & =A_{0}\left[\begin{array}{c}
w_{-n_{0}, e} \\
\vdots \\
w_{-n_{0}, e}
\end{array}\right]+B_{0,1} v_{e}, \\
0 & =\lambda_{k} w_{k, e}+a_{k} v_{e}, \quad|k| \geqslant n_{0}+1, \\
0 & =\sum_{k \in \mathbb{Z}} w_{k, e}\left(e_{k}^{1}\right)^{\prime}(0) .
\end{aligned}
$$

In particular $\left(\lambda_{k} w_{k, e}\right)_{k \in \mathbb{Z}}$ is square summable and thus $w_{e} \in D(\mathcal{A})$. The developments of Subsection 3.3 show that the above system is equivalent to

$$
\mathcal{A} w_{e}+a v_{e}=0
$$




$$
\left(w_{e}^{1}\right)^{\prime}(0)=0
$$

with $w_{e}=\left(w_{e}^{1}, w_{e}^{2}\right)$. By expanding the former identity, we first have that $\left(w_{e}^{1}\right)^{\prime \prime}+f^{\prime}\left(y_{e}\right) w_{e}^{1}=0$ with $w_{e}^{1}(0)=\left(w_{e}^{1}\right)^{\prime}(0)=0$ and thus, by Cauchy uniqueness, $w_{e}^{1}=0$. We also have $w_{e}^{2}=\frac{-x}{\alpha L} v_{e}$ with $v_{e}=-\alpha w_{e}^{2}(L)=\left(w_{e}^{1}\right)^{\prime}(L)=0$, and thus $w_{e}^{2}=0$. This yields $w_{e}=0$, which shows that $w_{k, e}=0$ for every $k \in \mathbb{Z}$. Hence the kernel of $T$ is reduced to $\{0\}$, which concludes the proof.

The result of Lemma 3 ensures the existence of a gain $K \in \mathbb{R}^{1 \times\left(2 n_{0}+3\right)}$ such that $A_{K}=A+B K$ is Hurwitz. Then, we can set the state feedback

$$
v_{d}(t)=\dot{v}(t)=K X(t),
$$

which yields the following closed-loop system dynamics:

$$
\begin{aligned}
& \dot{X}(t)=A_{K} X(t)+\Gamma(t), \\
& \dot{w}_{k}(t)=\lambda_{k} w_{k}(t)+a_{k} v(t)+b_{k} v_{d}(t)+r_{k}(t), \quad|k| \geqslant n_{0}+1 .
\end{aligned}
$$

The objective of the remainder of the paper is to assess the local stability of the closed-loop system, as well as the study of the tracking performance.

\section{Stability and set point regulation assessment}

\subsection{Stability analysis}

The main stability result of this paper is stated in the following theorem.

Theorem 1. There exist $\kappa \in(0,1)$ and $\bar{C}_{1}, \delta>0$ such that, for any $\eta \in[0,1)$, there exists $\bar{C}_{2}>0$ such that, for any initial condition satisfying

$$
\|W(0)\|_{\mathcal{H}}^{2}+|\xi(0)|^{2}+|v(0)|^{2} \leqslant \delta
$$

and any continuously differentiable reference input $z_{r}$ with

$$
\left\|z_{r}\right\|_{L^{\infty}\left(\mathbb{R}_{+}\right)}^{2} \leqslant \delta
$$

the classical solutions of (8) with control law (30) is well defined on $\mathbb{R}_{+}$and satisfies

$$
\left\|w^{1}(t, \cdot)\right\|_{L^{\infty}(0, L)}<1
$$

and

$$
\begin{aligned}
\|W(t)\|_{\mathcal{H}}^{2}+|\xi(t)|^{2}+|v(t)|^{2} \leqslant & \bar{C}_{1} e^{-2 \kappa t}\left(\|W(0)\|_{\mathcal{H}}^{2}+|\xi(0)|^{2}+|v(0)|^{2}\right) \\
& +\bar{C}_{2} \sup _{0 \leqslant s \leqslant t} e^{-2 \eta \kappa(t-s)}\left|z_{r}(s)\right|^{2} .
\end{aligned}
$$

for all $t \geqslant 0$.

Remark 7. In the particular case of a linear function $f$, which implies that the residual term (9) is identically zero, the exponential stability result (33) stated by Theorem 1 is global.

Remark 8. The result of Theorem 1 ensures the stability of the closed-loop system in $(w, \xi)$ coordinates. This immediately induces the stability of the closed-loop system in its original coordinates because, from (7), we have

$$
\left\|\left(y_{\delta}(t, \cdot), \frac{\partial y_{\delta}}{\partial t}(t, \cdot)\right)\right\|_{\mathcal{H}} \leqslant\|W(t)\|_{\mathcal{H}}+\left\|\left(0, \frac{(\cdot)}{\alpha L} v(t)\right)\right\|_{\mathcal{H}}
$$




$$
\leqslant\|W(t)\|_{\mathcal{H}}+\frac{1}{\alpha} \sqrt{\frac{L}{3}}|v(t)|
$$

and, from (24),

$$
\begin{aligned}
|\zeta(t)| & \leqslant|\xi(t)|+\sum_{|k| \geqslant n_{0}+1}\left|\frac{\left(e_{k}^{1}\right)^{\prime}(0)}{\lambda_{k}} w_{k}(t)\right| \\
& \leqslant|\xi(t)|+\sqrt{\sum_{|k| \geqslant n_{0}+1}\left|\frac{\left(e_{k}^{1}\right)^{\prime}(0)}{\lambda_{k}}\right|^{2}} \sqrt{\sum_{|k| \geqslant n_{0}+1}\left|w_{k}(t)\right|^{2}} \\
& \leqslant|\xi(t)|+\sqrt{\frac{1}{m_{R}} \sum_{|k| \geqslant n_{0}+1}\left|\frac{\left(e_{k}^{1}\right)^{\prime}(0)}{\lambda_{k}}\right|^{2}}\|W(t)\|_{\mathcal{H}}
\end{aligned}
$$

where we recall that $\left(e_{k}^{1}\right)^{\prime}(0)=O(1)$ and $\left|\lambda_{k}\right| \sim k \pi / L$ when $|k| \rightarrow+\infty$.

Proof of Theorem 1. Let $M>3\left(\|a\|_{\mathcal{H}}^{2}+\|b\|_{\mathcal{H}}^{2}\|K\|^{2}\right) / m_{R}$ be given, where we recall that $a, b \in \mathcal{H}$ are defined by (14) and the constant $m_{R}>0$ is as provided by Definition 2 . We introduce for all $t \geqslant 0$

$$
V(t)=M X(t)^{\top} P X(t)+\frac{1}{2} \sum_{|k| \geqslant n_{0}+1}\left|w_{k}(t)\right|^{2},
$$

where $P$ is a symmetric definite positive matrix such that $A_{K}^{\top} P+P A_{K}=-I$. Then we obtain from (31) that

$$
\begin{aligned}
\dot{V}(t)= & M X(t)^{\top}\left\{A_{K}^{\top} P+P A_{K}\right\} X(t)+M\left\{\Gamma(t)^{\top} P X(t)+X(t)^{\top} P \Gamma(t)\right\} \\
& +\sum_{|k| \geqslant n_{0}+1} \operatorname{Re} \lambda_{k}\left|w_{k}(t)\right|^{2}+\sum_{|k| \geqslant n_{0}+1} \operatorname{Re}\left\{\overline{w_{k}(t)}\left(a_{k} v(t)+b_{k} v_{d}(t)+r_{k}(t)\right)\right\} \\
\leqslant & -M\|X(t)\|^{2}-\sum_{|k| \geqslant n_{0}+1}\left|w_{k}(t)\right|^{2}+2 M\|P\|\|X(t)\|\|\Gamma(t)\| \\
& +\sum_{|k| \geqslant n_{0}+1}\left|w_{k}(t)\right|\left(\left|a_{k}\right||v(t)|+\left|b_{k}\right|\left|v_{d}(t)\right|+\left|r_{k}(t)\right|\right)
\end{aligned}
$$

where we have used that $\operatorname{Re} \lambda_{k}<-1$ for all $|k| \geqslant n_{0}+1$. Using now Young's inequality, we infer that

$$
2 M\|P\|\|X(t)\|\|\Gamma(t)\| \leqslant \frac{M}{2}\|X(t)\|^{2}+2 M\|P\|^{2}\|\Gamma(t)\|^{2}
$$

and

$$
\begin{aligned}
& \sum_{|k| \geqslant n_{0}+1}\left|w_{k}(t)\right|\left(\left|a_{k}\right||v(t)|+\left|b_{k}\right|\left|v_{d}(t)\right|+\left|r_{k}(t)\right|\right) \\
& \quad \leqslant \frac{1}{2} \sum_{|k| \geqslant n_{0}+1}\left|w_{k}(t)\right|^{2}+\frac{3}{2} \sum_{|k| \geqslant n_{0}+1}\left(\left|a_{k}\right|^{2}|v(t)|^{2}+\left|b_{k}\right|^{2}\left|v_{d}(t)\right|^{2}+\left|r_{k}(t)\right|^{2}\right) \\
& \quad \leqslant \frac{1}{2} \sum_{|k| \geqslant n_{0}+1}\left|w_{k}(t)\right|^{2}+\frac{3\|a\|_{\mathcal{H}}^{2}}{2 m_{R}}|v(t)|^{2}+\frac{3\|b\|_{\mathcal{H}}^{2}}{2 m_{R}}\left|v_{d}(t)\right|^{2}+\frac{3}{2 m_{R}}\|R(t, \cdot)\|_{\mathcal{H}}^{2} \\
& \quad \leqslant \frac{1}{2} \sum_{|k| \geqslant n_{0}+1}\left|w_{k}(t)\right|^{2}+\frac{3\left(\|a\|_{\mathcal{H}}^{2}+\|b\|_{\mathcal{H}}^{2}\|K\|^{2}\right)}{2 m_{R}}\|X(t)\|^{2}+\frac{3}{2 m_{R}}\|R(t, \cdot)\|_{\mathcal{H}}^{2}
\end{aligned}
$$


where we have used (30) and the fact that $v(t)$ is the first component of $X(t)$. Thus, we obtain that

$$
\begin{aligned}
\dot{V}(t) \leqslant & -\left\{\frac{M}{2}-\frac{3\left(\|a\|_{\mathcal{H}}^{2}+\|b\|_{\mathcal{H}}^{2}\|K\|^{2}\right)}{2 m_{R}}\right\}\|X(t)\|^{2}-\frac{1}{2} \sum_{|k| \geqslant n_{0}+1}\left|w_{k}(t)\right|^{2} \\
& +2 M\|P\|^{2}\|\Gamma(t)\|^{2}+\frac{3}{2 m_{R}}\|R(t, \cdot)\|_{\mathcal{H}}^{2} .
\end{aligned}
$$

We evaluate the two last terms of the above inequality. Recalling that $R_{1}(t), \gamma(t)$, and $\Gamma(t)$ are defined by (23a), (25c), and (26b), respectively, we have

$$
\begin{aligned}
\|\Gamma(t)\|^{2} & =\left\|R_{1}(t)\right\|^{2}+|\gamma(t)|^{2} \\
& =\sum_{|k| \leqslant n_{0}}\left|r_{k}(t)\right|^{2}+\left|z_{r}(t)+\sum_{|k| \geqslant n_{0}+1} \frac{\left(e_{k}^{1}\right)^{\prime}(0)}{\lambda_{k}} r_{k}(t)\right|^{2} \\
& \leqslant \sum_{|k| \leqslant n_{0}}\left|r_{k}(t)\right|^{2}+2\left|z_{r}(t)\right|^{2}+2 \sum_{|k| \geqslant n_{0}+1}\left|\frac{\left(e_{k}^{1}\right)^{\prime}(0)}{\lambda_{k}}\right|^{2} \sum_{|k| \geqslant n_{0}+1}\left|r_{k}(t)\right|^{2} \\
& \leqslant C_{0}^{2}\|R(t, \cdot)\|_{\mathcal{H}}^{2}+2\left|z_{r}(t)\right|^{2}
\end{aligned}
$$

with $C_{0}>0$ defined by

$$
C_{0}^{2}=\frac{1}{m_{R}} \max \left(1,2 \sum_{|k| \geqslant n_{0}+1}\left|\frac{\left(e_{k}^{1}\right)^{\prime}(0)}{\lambda_{k}}\right|^{2}\right)<+\infty .
$$

We now evaluate $\|R(t, \cdot)\|_{\mathcal{H}}^{2}=\int_{0}^{L}|r(t, x)|^{2} \mathrm{~d} x$ where we recall that $r(t, x)$ is defined by (9). Let $\epsilon \in$ $(0,1)$ to be determined. Let $C_{I} \geqslant 0$ be the maximum of $\left|f^{\prime \prime}\right|$ over the range $\left[\min y_{e}-1, \max y_{e}+1\right]$. Thus, assuming that

$$
\left\|w^{1}(t, \cdot)\right\|_{L^{\infty}(0, L)} \leqslant \epsilon<1,
$$

we obtain that $|r(t, x)| \leqslant C_{I}\left|w^{1}(t, x)\right|^{2}$ and thus

$$
\begin{aligned}
\|R(t, \cdot)\|_{\mathcal{H}}^{2} & =\int_{0}^{L}|r(t, x)|^{2} \mathrm{~d} x \\
& \leqslant C_{I}^{2} \int_{0}^{L}\left|w^{1}(t, x)\right|^{4} \mathrm{~d} x \\
& \leqslant \epsilon^{2} C_{I}^{2}\left\|w^{1}(t, \cdot)\right\|_{L^{2}(0, L)}^{2} \\
& \leqslant \epsilon^{2} L^{2} C_{I}^{2}\|W(t)\|_{\mathcal{H}}^{2} \\
& \leqslant \epsilon^{2} M_{R} L^{2} C_{I}^{2} \sum_{k \in \mathbb{Z}}\left|w_{k}(t)\right|^{2} \\
& \leqslant \epsilon^{2} C_{1}^{2}\left\{\|X(t)\|^{2}+\sum_{|k| \geqslant n_{0}+1}\left|w_{k}(t)\right|^{2}\right\}
\end{aligned}
$$

where the third inequality follows from Poincaré inequality, the fourth inequality follows from Definition 2, and with the constant $C_{1} \geqslant 0$ defined by $C_{1}^{2}=M_{R} L^{2} C_{I}^{2}$. We deduce from (35-36) and, under the a priori estimate (37), (39) that

$$
\dot{V}(t) \leqslant-\left\{\frac{M}{2}-\frac{3\left(\|a\|_{\mathcal{H}}^{2}+\|b\|_{\mathcal{H}}^{2}\|K\|^{2}\right)}{2 m_{R}}-\epsilon^{2} C_{1}^{2}\left(2 M\|P\|^{2} C_{0}^{2}+\frac{3}{2 m_{R}}\right)\right\}\|X(t)\|^{2}
$$




$$
\begin{aligned}
& -\frac{1}{2}\left\{\left(1-2 \epsilon^{2} C_{1}^{2}\left(2 M\|P\|^{2} C_{0}^{2}+\frac{3}{2 m_{R}}\right)\right\} \sum_{|k| \geqslant n_{0}+1}\left|w_{k}(t)\right|^{2}\right. \\
& +4 M\|P\|^{2}\left|z_{r}(t)\right|^{2} .
\end{aligned}
$$

With $M>3\left(\|a\|_{\mathcal{H}}^{2}+\|b\|_{\mathcal{H}}^{2}\|K\|^{2}\right) / m_{R}$ and by selecting $\epsilon \in(0,1)$ small enough (independently of the initial conditions and the reference signal $z_{r}$ ), we obtain the existence of constants $\kappa, C_{2}>0$ such that, under the a priori estimate (37),

$$
\dot{V}(t) \leqslant-2 \kappa V(t)+C_{2}\left|z_{r}(t)\right|^{2} .
$$

Let $\eta \in[0,1)$ be arbitrary. Assuming that the a priori estimate (37) holds on $[0, t]$ for some $t>0$, we obtain that

$$
\begin{aligned}
V(t) & \leqslant e^{-2 \kappa t} V(0)+C_{2} \int_{0}^{t} e^{-2 \kappa(t-s)}\left|z_{r}(s)\right|^{2} \mathrm{~d} s \\
& \leqslant e^{-2 \kappa t} V(0)+\frac{C_{2}}{2 \kappa(1-\eta)} \sup _{0 \leqslant s \leqslant t} e^{-2 \eta \kappa(t-s)}\left|z_{r}(s)\right|^{2} .
\end{aligned}
$$

Denoting by $\lambda_{m}(P)>0$ and $\lambda_{M}(P)>0$ the smallest and largest eigenvalues of the symmetric definite positive matrix $P$, we now note from (34) that

$$
\begin{aligned}
V(0) & \leqslant M \lambda_{M}(P)\|X(0)\|^{2}+\frac{1}{2} \sum_{|k| \geqslant n_{0}+1}\left|w_{k}(0)\right|^{2} \\
& \leqslant C_{3}\left(\|W(0)\|_{\mathcal{H}}^{2}+|\xi(0)|^{2}+|v(0)|^{2}\right)
\end{aligned}
$$

for some constant $C_{3}>0$ and

$$
\begin{aligned}
V(t) & \geqslant M \lambda_{m}(P)\|X(t)\|^{2}+\frac{1}{2} \sum_{|k| \geqslant n_{0}+1}\left|w_{k}(t)\right|^{2} \\
& \geqslant C_{4}\left(\|W(t)\|_{\mathcal{H}}^{2}+|\xi(t)|^{2}+|v(t)|^{2}\right)
\end{aligned}
$$

for some constant $C_{4}>0$. Thus, assuming that the a priori estimate (37) holds over $[0, t]$ for some $t>0$, we deduce from the three above estimates the existence of constants $\bar{C}_{1}, \bar{C}_{2}>0$ such that

$$
\begin{aligned}
\|W(t)\|_{\mathcal{H}}^{2}+|\xi(t)|^{2}+|v(t)|^{2} \leqslant & \bar{C}_{1} e^{-2 \kappa t}\left(\|W(0)\|_{\mathcal{H}}^{2}+|\xi(0)|^{2}+|v(0)|^{2}\right) \\
& +\bar{C}_{2} \sup _{0 \leqslant s \leqslant t} e^{-2 \eta \kappa(t-s)}\left|z_{r}(s)\right|^{2} .
\end{aligned}
$$

To conclude, let us note that

$$
\left\|w^{1}(t, \cdot)\right\|_{L^{\infty}(0, L)} \leqslant \sqrt{L}\left\|\left(w^{1}\right)^{\prime}(t, \cdot)\right\|_{L^{2}(0, L)} \leqslant \sqrt{L}\|W(t)\|_{\mathcal{H}} .
$$

Hence, if the initial condition is selected such that

$$
\|W(0)\|_{\mathcal{H}}^{2}+|\xi(0)|^{2}+|v(0)|^{2} \leqslant \frac{\epsilon^{2}}{2 L} \min \left(1, \frac{1}{2 \bar{C}_{1}}\right)
$$

which in particular ensures that $\left\|w^{1}(0, \cdot)\right\|_{L^{\infty}(0, L)} \leqslant \epsilon / \sqrt{2}<\epsilon$, and the reference input is chosen such that

$$
\left\|z_{r}\right\|_{L^{\infty}(0, L)}^{2} \leqslant \frac{\epsilon^{2}}{4 L \bar{C}_{2}}
$$

it is readily checked based on (40) that the a priori estimate (37) holds for all $t \geqslant 0$. In this case, the stability estimate (40) holds for all $t \geqslant 0$. 


\subsection{Set point regulation}

We are now in position to assess the set point regulation of the left Neumann trace.

Theorem 2. Let $\kappa \in(0,1), \delta>0$, and $\eta \in[0,1)$ be as provided by Theorem 1. There exist constants $\bar{C}_{3}, \bar{C}_{4}>0$ such that, for any initial condition satisfying

$$
\|W(0)\|_{\mathcal{H}}^{2}+|\xi(0)|^{2}+|v(0)|^{2} \leqslant \delta
$$

and any continuously differentiable reference input $z_{r}$ with

$$
\left\|z_{r}\right\|_{L^{\infty}\left(\mathbb{R}_{+}\right)}^{2} \leqslant \delta
$$

the classical solutions of (8) satisfies

$$
\begin{aligned}
\left|\left(w^{1}\right)^{\prime}(t, 0)-z_{r}(t)\right| \leqslant & \bar{C}_{3} e^{-\kappa t}\left\{\|W(0)\|_{\mathcal{H}}+|\xi(0)|+|v(0)|+\|\mathcal{A} W(0)\|_{\mathcal{H}}\right\} \\
& +\bar{C}_{4} \sup _{0 \leqslant s \leqslant t} e^{-\eta \kappa(t-s)}\left|z_{r}(s)\right|
\end{aligned}
$$

for all $t \geqslant 0$.

Remark 9. In particular, $z_{r}(t) \rightarrow 0$ implies $\left(w^{1}\right)^{\prime}(t, 0) \rightarrow 0$, i.e. $z(t) \rightarrow z_{e}$, which achieves the desired set point reference tracking.

Before proceeding with the proof of Theorem 2, we first derive an estimate of $\left\|\frac{\mathrm{d} R}{\mathrm{~d} t}(t, \cdot)\right\|_{\mathcal{H}}$. To do so, we assume that the assumptions and conclusions of Theorem 1 apply. Following up with Remark 4, we have that $\frac{\mathrm{d} R}{\mathrm{~d} t}=\left(0, \frac{\mathrm{d} r}{\mathrm{~d} t}\right)$ with

$$
\frac{\mathrm{d} r}{\mathrm{~d} t}(t, \cdot)=\left[w^{2}(t, \cdot)+\frac{(\cdot)}{\alpha L} v(t)\right] \int_{y_{e}}^{w^{1}(t, \cdot)+y_{e}} f^{\prime \prime}(s) \mathrm{d} s .
$$

Using (32) and the estimate $\left|f^{\prime \prime}\right| \leqslant C_{I}$ on the range $\left[\min y_{e}-1\right.$, max $\left.y_{e}+1\right]$, we deduce that

$$
\begin{aligned}
\left\|\frac{\mathrm{d} R}{\mathrm{~d} t}(t, \cdot)\right\|_{\mathcal{H}}^{2} & =\left\|\frac{\mathrm{d} r}{\mathrm{~d} t}(t, \cdot)\right\|_{L^{2}(0, L)}^{2} \\
& \leqslant \int_{0}^{L}\left|w^{2}(t, x)+\frac{x}{\alpha L} v(t)\right|^{2}\left|\int_{y_{e}(x)}^{w^{1}(t, x)+y_{e}(x)}\right| f^{\prime \prime}(s)|\mathrm{d} s|^{2} \mathrm{~d} x \\
& \leqslant 2 C_{I}^{2}\left\{\left\|w^{2}(t)\right\|_{L^{2}(0, L)}^{2}+\frac{L}{3 \alpha^{2}}|v(t)|^{2}\right\} \\
& \leqslant 2 C_{I}^{2}\left\{\|W(t)\|_{\mathcal{H}}^{2}+\frac{L}{3 \alpha^{2}}|v(t)|^{2}\right\}
\end{aligned}
$$

We are now in position to complete the proof of Theorem 2.

Proof of Theorem 2. We fix an integer $N \geqslant n_{0}$ and a constant $\gamma>0$ such that ${ }^{2} \operatorname{Re} \lambda_{k} \leqslant$ $-\gamma<-\kappa<0$ for all $|k| \geqslant N+1$. Then we have from (19) that

$$
\begin{aligned}
& \left|\left(w^{1}\right)^{\prime}(t, 0)-z_{r}(t)\right| \\
& \quad \leqslant \sum_{k \in \mathbb{Z}}\left|w_{k}(t)\right|\left|\left(e_{k}^{1}\right)^{\prime}(0)\right|+\left|z_{r}(t)\right|
\end{aligned}
$$

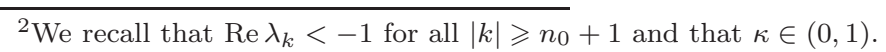




$$
\begin{aligned}
\leqslant & \sum_{|k| \leqslant N}\left|w_{k}(t)\right|\left|\left(e_{k}^{1}\right)^{\prime}(0)\right|+\sum_{|k| \geqslant N+1}\left|\lambda_{k} w_{k}(t)\right| \frac{\left(e_{k}^{1}\right)^{\prime}(0)}{\lambda_{k}}|+| z_{r}(t) \mid \\
\leqslant & \sqrt{\sum_{|k| \leqslant N}\left|\left(e_{k}^{1}\right)^{\prime}(0)\right|^{2}} \sqrt{\sum_{|k| \leqslant N}\left|w_{k}(t)\right|^{2}} \\
& +\sqrt{\sum_{|k| \geqslant N+1}\left|\frac{\left(e_{k}^{1}\right)^{\prime}(0)}{\lambda_{k}}\right|^{2}} \sqrt{\sum_{|k| \geqslant N+1}\left|\lambda_{k} w_{k}(t)\right|^{2}}+\left|z_{r}(t)\right| \\
\leqslant & \sqrt{\frac{1}{m_{R}} \sum_{|k| \leqslant N}\left|\left(e_{k}^{1}\right)^{\prime}(0)\right|^{2}} \mid W(t) \|_{\mathcal{H}} \\
& +\sqrt{\sum_{|k| \geqslant N+1}\left|\frac{\left(e_{k}^{1}\right)^{\prime}(0)}{\lambda_{k}}\right|^{2}} \sqrt{\sum_{|k| \geqslant N+1}\left|\lambda_{k} w_{k}(t)\right|^{2}}+\left|z_{r}(t)\right|
\end{aligned}
$$

Based on (33), we only need to evaluate the term $\sum_{|k| \leqslant N}\left|\lambda_{k} w_{k}(t)\right|^{2}$. We have from (31) that, for all $|k| \geqslant N+1 \geqslant n_{0}+1$,

$$
\begin{aligned}
\lambda_{k} w_{k}(t)= & e^{\lambda_{k} t} \lambda_{k} w_{k}(0)+\int_{0}^{t} \lambda_{k} e^{\lambda_{k}(t-s)}\left\{a_{k} v(s)+b_{k} v_{d}(s)+r_{k}(s)\right\} \mathrm{d} s \\
= & e^{\lambda_{k} t} \lambda_{k} w_{k}(0)-\left\{a_{k} v(t)+b_{k} v_{d}(t)+r_{k}(t)\right\} \\
& +e^{\lambda_{k} t}\left\{a_{k} v(0)+b_{k} v_{d}(0)+r_{k}(0)\right\} \\
& +\int_{0}^{t} e^{\lambda_{k}(t-s)}\left\{a_{k} v_{d}(s)+b_{k} \dot{v}_{d}(s)+\dot{r}_{k}(s)\right\} \mathrm{d} s .
\end{aligned}
$$

We have from (30-31) that $v_{d}(t)=K X(t)$ and $\dot{v}_{d}(t)=K A_{K} X(t)+K \Gamma(t)$ with

$$
\begin{aligned}
\|X(t)\|^{2} & \leqslant \frac{1}{m_{R}}\|W(t)\|_{\mathcal{H}}^{2}+|\xi(t)|^{2}+|v(t)|^{2}, \\
\|\Gamma(t)\|^{2} & \leqslant L^{2} C_{0}^{2} C_{I}^{2}\|W(t)\|_{\mathcal{H}}^{2}+2\left|z_{r}(t)\right|^{2}
\end{aligned}
$$

where the second inequality follows from (36) and (38). For ease of notation, we define CI = $\sqrt{\|W(0)\|_{\mathcal{H}}^{2}+|\xi(0)|^{2}+|v(0)|^{2}}$. Using $\gamma>\kappa>\eta \kappa \geqslant 0$, we have from (33) that

$$
\begin{aligned}
& \left|\int_{0}^{t} e^{\lambda_{k}(t-s)} v_{d}(s) \mathrm{d} s\right| \\
& \leqslant\|K\| \int_{0}^{t} e^{-\gamma(t-s)}\|X(s)\| \mathrm{d} s \\
& \leqslant\|K\| \max \left(1, m_{R}^{-1 / 2}\right) e^{-\gamma t} \int_{0}^{t} e^{\gamma s}\left\{\sqrt{\bar{C}_{1}} e^{-\kappa s} \mathrm{CI}+\sqrt{\bar{C}_{2}} \sup _{0 \leqslant \tau \leqslant s} e^{-\eta \kappa(s-\tau)}\left|z_{r}(\tau)\right|\right\} \mathrm{d} s \\
& \leqslant C_{5} e^{-\kappa t} \mathrm{CI}+C_{5} \sup _{0 \leqslant \tau \leqslant t} e^{-\eta \kappa(t-\tau)}\left|z_{r}(\tau)\right|
\end{aligned}
$$

for some constant $C_{5}>0$ and, similarly,

$$
\left|\int_{0}^{t} e^{\lambda_{k}(t-s)} \dot{v}_{d}(s) \mathrm{d} s\right| \leqslant C_{6} e^{-\kappa t} \mathrm{CI}+C_{6} \sup _{0 \leqslant \tau \leqslant t} e^{-\eta \kappa(t-\tau)}\left|z_{r}(\tau)\right|
$$


for some constant $C_{6}>0$. Finally, we also have, for $-\gamma<-\tilde{\kappa}<-\kappa<0$,

$$
\begin{aligned}
\left|\int_{0}^{t} e^{\lambda_{k}(t-s)} \dot{r}_{k}(s) \mathrm{d} s\right| & \leqslant \int_{0}^{t} e^{-\gamma(t-s)}\left|\dot{r}_{k}(s)\right| \mathrm{d} s \\
& \leqslant \int_{0}^{t} e^{-(\gamma-\tilde{\kappa})(t-s)} \times e^{-\tilde{\kappa}(t-s)}\left|\dot{r}_{k}(s)\right| \mathrm{d} s \\
& \leqslant \sqrt{\int_{0}^{t} e^{-2(\gamma-\tilde{\kappa})(t-s)} \mathrm{d} s} \sqrt{\int_{0}^{t} e^{-2 \tilde{\kappa}(t-s)}\left|\dot{r}_{k}(s)\right|^{2} \mathrm{~d} s} \\
& \leqslant \sqrt{\frac{1}{2(\gamma-\tilde{\kappa})}} \sqrt{\int_{0}^{t} e^{-2 \tilde{\kappa}(t-s)}\left|\dot{r}_{k}(s)\right|^{2} \mathrm{~d} s}
\end{aligned}
$$

Taking the square on both sides of (45), using Young's inequality, substituting estimates (33), (38), and (46), and using the fact that $\sum_{|k| \geqslant N+1}\left|\left\langle z, f_{k}\right\rangle\right|^{2} \leqslant\|z\|_{\mathcal{H}}^{2} / m_{R}$ for all $z \in \mathcal{H}$, we infer the existence of a constant $C_{7}>0$ such that

$$
\begin{aligned}
\sum_{|k| \geqslant N+1}\left|\lambda_{k} w_{k}(t)\right|^{2} \leqslant & C_{7} e^{-2 \kappa t} \sum_{|k| \geqslant N+1}\left|\lambda_{k} w_{k}(0)\right|^{2} \\
& +C_{7} e^{-2 \kappa t} \mathrm{CI}^{2}+C_{7} \sup _{0 \leqslant \tau \leqslant t} e^{-2 \eta \kappa(t-\tau)}\left|z_{r}(\tau)\right|^{2} \\
& +C_{7} \int_{0}^{t} e^{-2 \tilde{\kappa}(t-s)}\left\|\frac{\mathrm{d} R}{\mathrm{~d} t}(s, \cdot)\right\|_{\mathcal{H}}^{2} \mathrm{~d} s
\end{aligned}
$$

where we recall that $\operatorname{Re} \lambda_{k} \leqslant-\gamma<-\kappa<0$ for all $|k| \geqslant N+1$. Noting that, for $|k| \geqslant N+1 \geqslant$ $n_{0}+1,\left\langle\mathcal{A} W(0), f_{k}\right\rangle_{\mathcal{H}}=\lambda_{k} w_{k}(0)$, we infer that $\sum_{|k| \geqslant N+1}\left|\lambda_{k} w_{k}(0)\right|^{2} \leqslant \sum_{k \in \mathbb{Z}}\left|\left\langle\mathcal{A} W(0), f_{k}\right\rangle_{\mathcal{H}}\right|^{2} \leqslant$ $\|\mathcal{A} W(0)\|_{\mathcal{H}}^{2} / m_{R}$. Then, based on (33) and (43), and as $\tilde{\kappa}>\kappa>0$, estimations similar to the ones reported in (46c-46d) show the existence of a constant $C_{8}>0$ such that

$$
\sum_{|k| \geqslant N+1}\left|\lambda_{k} w_{k}(t)\right|^{2} \leqslant C_{8} e^{-2 \kappa t}\left\{\mathrm{CI}^{2}+\|\mathcal{A} W(0)\|_{\mathcal{H}}^{2}\right\}+C_{8} \sup _{0 \leqslant \tau \leqslant t} e^{-2 \eta \kappa(t-\tau)}\left|z_{r}(\tau)\right|^{2} .
$$

Substituting this latter estimate and (33) into (44), we obtain the existence of constants $\bar{C}_{3}, \bar{C}_{4}>0$ such that (41) holds.

\section{$5 \quad$ Numerical illustration}

For numerical illustration, we set $L=1, \alpha=1.1$, and we consider the nonlinear function $f(y)=y^{3}$, which leads to the boundary control system:

$$
\begin{aligned}
& \frac{\partial^{2} y}{\partial t^{2}}=\frac{\partial^{2} y}{\partial x^{2}}+y^{3}, \\
& y(t, 0)=0, \quad \frac{\partial y}{\partial x}(t, L)=u(t), \\
& y(0, x)=y_{0}(x), \quad \frac{\partial y}{\partial t}(0, x)=y_{1}(x),
\end{aligned}
$$

for $t>0$ and $x \in(0,1)$. Since $F(y)=\int_{0}^{y} f(s) \mathrm{d} s=y^{4} / 4 \rightarrow+\infty$ when $|y| \rightarrow+\infty$, it follows from Remark 1 the existence of a steady state $y_{e} \in \mathcal{C}^{2}([0, L])$ associated with any given value of the system output $z_{e} \in \mathbb{R}=y_{e}^{\prime}(0)$. We set $z_{e}=1.5$ and numerically compute the associated 


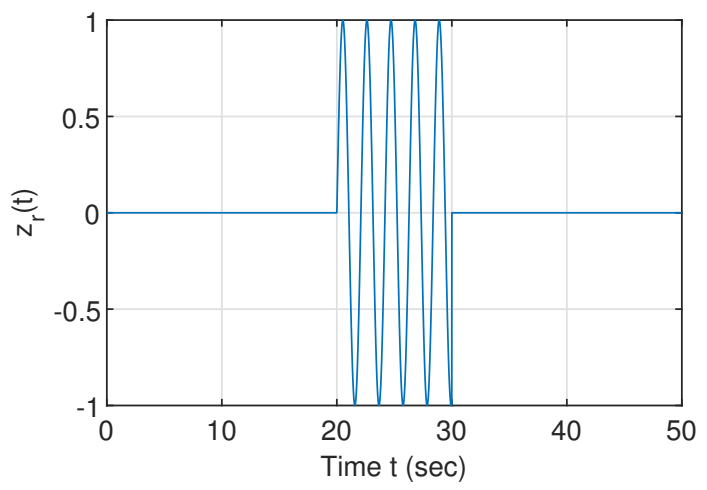

Figure 1: Signal $z_{r}(t)$

steady-state trajectory $y_{e}$, giving in particular the equilibrium control input $u_{e}=y_{e}^{\prime}(L) \approx 0.781$. In the absence of non linearity, i.e. $f=0$, it is known (see e.g. [38, Section 4]) that the eigenvalues of the operator $\mathcal{A}$ defined by (13) are given by

$$
\lambda_{k}=\frac{1}{2 L} \log \left(\frac{\alpha-1}{\alpha+1}\right)+i \frac{k \pi}{L} .
$$

These values are used as initial guesses to determine the eigenvalues $\lambda_{k}$ and the associated eigenvectors $e_{k}$ of the operator $\mathcal{A}$ in the presence of the non linearity $f(y)=y^{3}$ by using a shooting method. We obtain one unstable eigenvalue $\lambda_{0} \approx 0.326$ while all other eigenvalues are stable with a real part less than 1 . The feedback gain is computed to place the poles of the truncated model at $-0.5,-1$, and -1.5 .

For numerical simulations, we select the initial condition $W(0, x)=\left(\frac{2 \alpha}{5} x,-\frac{2}{5 L} x\right) \in D(\mathcal{A})$ and the signal $z_{r}$ as depicted in Fig. 1. The adopted numerical scheme is the modal approximation of the infinite-dimensional system using its first 10 modes. The time domain evolution of the state of the closed-loop system, the regulated output, and the command input, are depicted in Fig. 2. The simulation results are compliant with the theoretical predictions.

\section{Conclusion and open issues}

In this paper we have investigated the proportional integral (PI) boundary regulation control of the left Neumann trace of a one-dimensional semilinear wave equation. Our control strategy combines a traditional velocity feedback and the design of an auxiliary control law performed on a finite-dimensional (spectrally) truncated model.

A number of open issues and potential directions for further research emerge from this study, that we list and comment hereafter.

\subsection{Other controls and outputs}

Other controls In this paper, we have considered a Neumann boundary control, on the right of the interval. Of course, we could have taken this control on the left, or even on both sides. While the proposed PI procedure seems to be extendable to the cases of a Robin boundary control, 


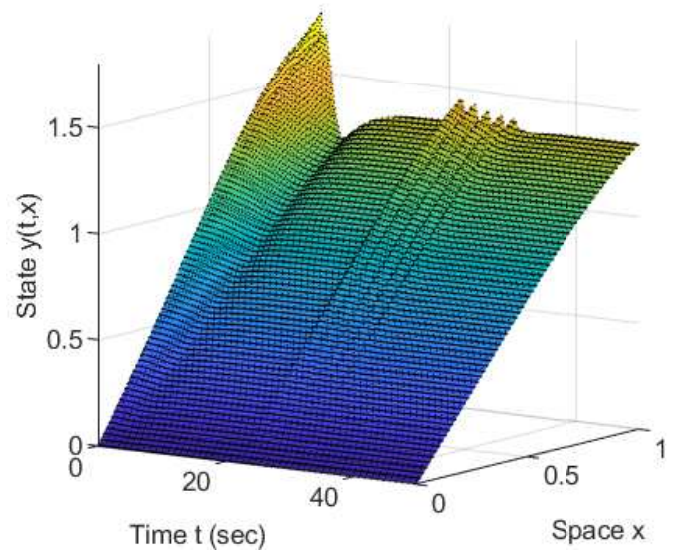

(a) State $y(t, x)$

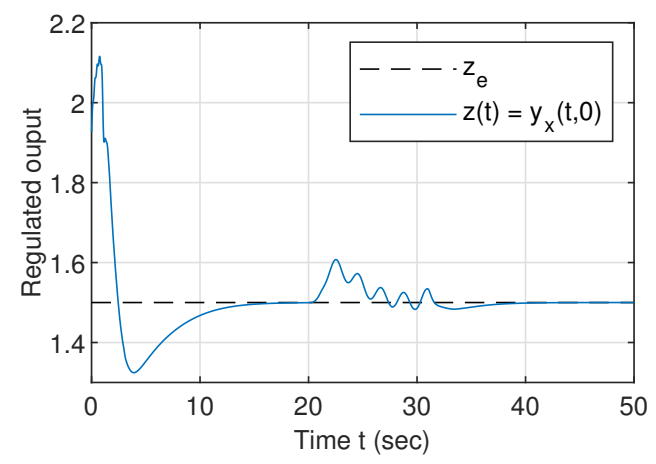

(c) Regulated output $z(t)=\frac{\partial y}{\partial x}(t, 0)$

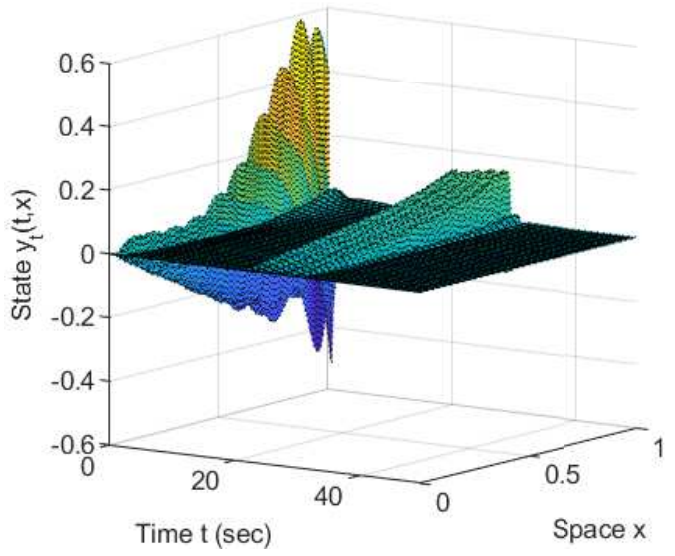

(b) State $\frac{\partial y}{\partial t}(t, x)$

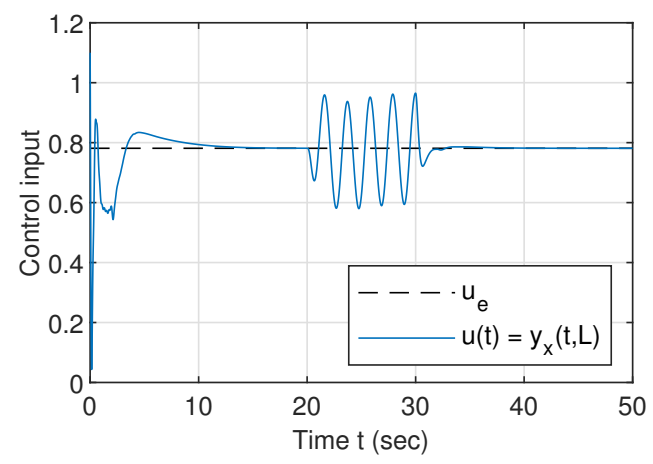

(d) Control input $u(t)=\frac{\partial y}{\partial x}(t, L)$

Figure 2: Time domain evolution of the state of the closed-loop system 
of mixed Dirichlet-Neumann boundary controls, and of a distributed (i.e., internal) control input (note that, in this case, the control operator is bounded), the case of a Dirichlet boundary control seems much more challenging and remains open.

Other outputs We have selected the regulated ouput as the Neumann trace. However, one might be interested in regulating other types of outputs. This includes, for example, the Dirichlet trace at the left boundary or the value of $y$ either at a specific location or on a subset of the spatial domain. It is not clear whether or not the reported PI control design procedure could be extended to these different settings.

\subsection{The general multi-dimensional case}

In this paper we have focused on the one-dimensional wave equation, which is already quite challenging. The multi-dimensional case is completely open, in particular because, except in very particular cases (like the two-dimensional disk for instance), we do not have any Riesz basis formed by generalized eigenvectors of the underlying unbounded operator. Indeed, the Riesz basis property is essentially a one-dimensional property.

Hence, in the multi-dimensional case, a first challenging difficulty that must be addressed is to replace the Riesz basis study by more abstract projection operators. On this issue, it seems that the recent work [18] provides very interesting line of research, although the authors of that reference deal with a parabolic equation with a selfadjoint operator and then one has a spectral decomposition and spectral projectors. For a wave equation, the underlying operator is not selfadjoint and is not even normal, which creates deep difficulties for the spectral study.

At present, we leave this issue completely open.

\subsection{Robustness with respect to perturbations}

Beyond the set-point regulation control of the system output, PI controllers are well known for their ability to provide, in general, a form of robustness with respect to external disturbances. The case of a continuously differentiable additive distributed disturbance $d(t, \cdot) \in L^{2}(0, L)$, yielding the dynamics

$$
\frac{\partial^{2} y}{\partial t^{2}}=\frac{\partial^{2} y}{\partial x^{2}}+f(y)+d,
$$

can be easily handled in our approach by merely embedding the contribution of the disturbance $d$ into the residual term $r$ given by (9). See also [29] for the case of a linear reaction-diffusion equation. A similar robustness issue would be to evaluate the impact of an additive boundary disturbance $p(t)$ applying to the control input:

$$
\frac{\partial y}{\partial x}(t, L)=u(t)+p(t) .
$$

However, comparing to the case of distributed perturbations, the study of the robustness of infinitedimensional systems with respect to boundary perturbations is generally much more challenging [33].

\subsection{Robustness with respect to the domain}

Another interesting robustness issue regarding the studied wave equation (1) concerns the robustness of the PI procedure with respect to uncertainties on the length $L>0$ of the domain. Indeed, in many engineering devices the value of $L$ is known only approximately; it may even happen that, 
along the process, the domain varies a bit. The robustness of the control strategy with respect to $L$ becomes then a major issue.

In our strategy, the value of the parameter $L$ directly impacts the vectors of the Riesz-basis $\left(e_{k}\right)_{k \in \mathbb{Z}}$. However, since the control strategy relies on a finite dimensional truncated model, only the perturbations on a finite number of vectors of the Riesz-basis have an impact on the control strategy. Hence, similarly to the study reported in [16], the Riesz basis property remains uniform with respect to small perturbations of $L>0$. This allows to easily derive a robustness result versus small variations of the length of the spatial domain, and thus to give a positive answer to the question of robustness with respect to $L$.

This result shows the power and advantage of the strategy developed here, based on spectral finite-dimensional truncations of the problem, which conveys robustness properties.

In the multi-dimensional case, the question of robustness with respect to the domain would certainly be much more difficult to address. It would be required to introduce an appropriate shape topology and then perform a kind of sensitivity analysis on the control design with respect to that topology.

\subsection{Robustness with respect to input and/or state delays}

In this section, we discuss the impact of possible input or state delays on the reported control strategy. As we explain below, we cannot expect any robustness of our strategy with respect to input delays, while the situation for state delays is expected to be more favourable.

Input delay It was shown in [29] for a 1-D reaction-diffusion equation that the PI controller design procedure can be augmented with a predictor component $[7,13,28]$ to handle constant control input delays. Such a strategy fails in the context of the wave equation studied in this paper. The reason is that, even for arbitrarily small $h>0$, the preliminary feedback

$$
u_{\delta}(t)=-\alpha \frac{\partial y_{\delta}}{\partial t}(t-h, L)
$$

might fail to stabilize the linear wave equation, yielding an infinite number of unstable modes. To illustrate this remark, let us consider the following linear wave equation:

$$
\begin{aligned}
& \frac{\partial^{2} y}{\partial t^{2}}=\frac{\partial^{2} y}{\partial x^{2}}-\beta y, \\
& y(t, 0)=0, \quad \frac{\partial y}{\partial x}(t, L)=-\alpha \frac{\partial y}{\partial t}(t-h, L),
\end{aligned}
$$

where $h>0$ is a constant delay, $\alpha>0$, and $\beta \in \mathbb{R}$. It was shown in [17] in the case $\alpha=1$ and $\beta=0$ that (48) admits an infinite number of unstable modes for some arbitrarily small values of the delay $h>0$. We elaborate here this remark by extending it to the case $\alpha>0$ and $\beta \in \mathbb{R}$. We proceed similarly to [17] by looking for solutions of (48) under the form $w(t, x)=e^{\lambda t} \sinh \left(\sqrt{\lambda^{2}+\beta} x\right)$ for some $\lambda \in \mathbb{C}$. It is easy to see that such a solution exists if and only if $\lambda \in \mathbb{C}$ satisfies

$$
\sqrt{\lambda^{2}+\beta} \cosh \left(\sqrt{\lambda^{2}+\beta} L\right)=-\alpha \lambda e^{-\lambda h} \sinh \left(\sqrt{\lambda^{2}+\beta} L\right) .
$$

We start by studying the special case $\beta=0$. Then the above identity becomes equivalent to:

$$
e^{\lambda h}=-\alpha \tanh (\lambda L) \text {. }
$$

Let $k \in \mathbb{Z}$ be arbitrarily fixed and select the input delay

$$
h=\frac{L}{k+1 / 2} \text {. }
$$


Let $\gamma>0$ be the only positive number such that

$$
e^{\gamma h}=\alpha \operatorname{coth}(\gamma L) \text {. }
$$

It is easily seen that $\lambda_{n}^{0}=\gamma+\frac{i}{L}\left(k+\frac{1}{2}\right)(4 n+1) \pi, n \in \mathbb{Z}$, are distinct solutions of (49) with $\operatorname{Re} \lambda_{n}=\gamma>0$. We now turn out attention onto the case $\beta \neq 0$. Let $h, \gamma>0$ be still given by (50-51). We introduce the open set $A=\{\lambda \in \mathbb{C}: 0<\operatorname{Re} z<2 \gamma,|\lambda|>\sqrt{\beta}\}$. We define for $\lambda \in A$ the holomorphic functions

$$
\begin{aligned}
& f(\lambda)=\lambda \cosh (\lambda L)+\alpha \lambda e^{-\lambda h} \sinh (\lambda L), \\
& g(\lambda)=\sqrt{\lambda^{2}+\beta} \cosh \left(\sqrt{\lambda^{2}+\beta} L\right)+\alpha \lambda e^{-\lambda h} \sinh \left(\sqrt{\lambda^{2}+\beta} L\right),
\end{aligned}
$$

with $\sqrt{\lambda^{2}+\beta}=\lambda \exp \left(\frac{1}{2} \log \left(1+\frac{\beta}{\lambda^{2}}\right)\right)$, where $\log$ denotes the principal determination of logarithm. We have $f\left(\lambda_{n}^{0}\right)=0$ for all $n \in \mathbb{Z}$ and it can be observed that

$$
g(\lambda)=f(\lambda)+O(1), \quad \lambda \in A, \quad|\lambda| \rightarrow+\infty .
$$

For a given constant $R>0$ to be defined later, we consider the simple loop $\lambda_{n}:[0,2 \pi] \rightarrow \mathbb{C}$ defined by $\lambda_{n}(\theta)=\lambda_{n}^{0}+\frac{R e^{i \theta}}{n}, \theta \in[0,2 \pi]$. We consider an integer $N_{0} \geqslant 1$ such that $\lambda_{n}(\theta) \in A$ for all $|n| \geqslant N_{0}$ and all $\theta \in[0,2 \pi]$. Standard computations show that

$$
f\left(\lambda_{n}(\theta)\right)=(-1)^{k} i \lambda_{n}^{0} \frac{R e^{i \theta}}{n} \zeta \cosh (\gamma L)+O\left(\frac{1}{n}\right)
$$

when $|n| \rightarrow+\infty$, uniformly with respect to $\theta \in[0,2 \pi]$, where $\zeta=L+h \tanh (\gamma L)-L \tanh ^{2}(\gamma L)$. We note that $\zeta \neq 0$. Indeed, the condition $\zeta=0$ would imply that $\tanh (\gamma L)>0$ is the positive root of $L+h X-L X^{2}$, yielding the contradiction $\tanh (\gamma L)=\left(h+\sqrt{h^{2}+4 L^{2}}\right) /(2 L)>1+h /(2 L)>1$. We deduce that

$$
\left|f\left(\lambda_{n}(\theta)\right)\right| \sim C R
$$

when $|n| \rightarrow+\infty$, uniformly with respect to $\theta \in[0,2 \pi]$, where the constant $C=\frac{2(2 k+1) \pi|\zeta| \cosh (\gamma L)}{L} \neq$ 0 is independent of $R>0$. Hence, in view of (52-53) and by selecting $R>0$ large enough, we can apply Rouché's theorem for $|n| \geqslant N_{1}$ with $N_{1}>0$ large enough. This shows for any $|n| \geqslant N_{1}$ the existence of $\lambda_{n}^{\beta}=\lambda_{n}^{0}+O\left(\frac{1}{n}\right)$ such that $g\left(\lambda_{n}^{\beta}\right)=0$. Since $h>0$ defined by (50) can be made arbitrarily small by selecting $k$ arbitrarily large, we have shown the existence of arbitrarily small delays $h>0$ such that (48) admits an infinite number of unstable modes. Such an observation implies that the strategy reported in this paper cannot be successfully applied to the case of a delayed control input. Hence, the PI regulation control of (1) in the presence of a delay in the control input remains open.

State delay While, as discussed above, the case of a delay in the boundary control induces many difficulties (in particular, an infinite number of unstable modes), the case of a delay in the nonlinearity $f$ might be more favourable. More specifically, a possible research direction is concerned with the potential extension of the control strategy reported in this paper to the case of the wave equation

$$
\frac{\partial^{2} y}{\partial t^{2}}(t, x)=\frac{\partial^{2} y}{\partial x^{2}}(t, x)+f(y(t-h, x))
$$

where $h>0$ is a state-delay. Possible approaches include the use of either Lyapunov-Krasovskii functionals [25] or small gain arguments [30]. 


\subsection{Alternatives for control design}

In this paper, we have designed the control strategy, in particular, by using the pole shifting theorem (see in particular equation 30 in Subsection 3.4). Such a pole shifting is very natural considering the spectral approach that we have implemented: placing adequately the poles (of course, the unstable ones; but we can also shift to the left some of the stable ones in order to improve the stabilization properties) we are able to ensure some robustness properties, with respect to disturbances or with respect to the domain, as we have discussed previously.

One can wonder whether this is possible to design the control by other methods. For instance by the celebrated Riccati procedure (see, e.g., [24, 40, 44, 49]) applied to the truncated finitedimensional system. Considering the classical Linear Quadratic Riccati theory, let us denote by $u_{n}$ the optimal Riccati control (for some given weights) associated with the truncated system in dimension $n$. We do not make precise all notations but the framework is clear. The main question is: does $u_{n}$ converge to $u_{\infty}$ as $n \rightarrow+\infty$, where $u_{\infty}$ is the Riccati control of the complete system in infinite dimension (i.e., the PDE)?

We expect that such a convergence property is true at least in the parabolic case, i.e., for instance, for heat-like equations, or in the hyperbolic case with internal controls, i.e., for instance, for wave-like equations with distributed control. The hyperbolic case with boundary controls is probably much more difficult.

Adjacently, this discussion raises the problem of discussing the numerical efficiency of various control design approaches. It would be interesting to compare our approach, developed in the present paper, with other possible approaches and to compare their efficiency. One different approach that may come to our mind is backtepping design, which is well known to promote robustness properties (see, e.g., [26]), at least, for parabolic equations. It is not clear whether backstepping could be performed for the 1-D wave equation investigated in the present article.

\subsection{Controlling the output regulation by quasi-static deformations}

In the present work, we have dealt with the stabilization and regulation control in the vicinity of a given steady-state. In this section, we address the following question: considering that the system output has been regulated to a given steady-state, is it now possible to steer the system output to another steady-state?

More precisely, based on Definition 1, we denote by $\mathcal{S} \subset \mathcal{C}^{2}([0, L])$ the set of steady-states of (1) endowed with the $\mathcal{C}^{2}([0, L])$ topology. Let $y_{e, 1}, y_{e, 2} \in \mathcal{S}$ belonging to the same connected component of $\mathcal{S}$. Introducing for $i \in\{1,2\}$ the system output associated with the steady-state $y_{e, i}$ defined by

$$
z_{e, i}=\frac{\mathrm{d} y_{e, i}}{\mathrm{~d} x}(0),
$$

can we design a PI controller able to steer the system output from its initial value $z_{e, 1}$ (or, close to it) into any neighbourhood of $z_{e, 2}$ in finite time?

A way to address this issue is to design a PI controller by quasi-static deformation, as in $[15,16,39]$, along a path of steady outputs connecting $z_{e, 1}$ to $z_{e, 2}$. More precisely, since $y_{e, 1}, y_{e, 2} \in$ $\mathcal{S}$ are assumed to belong to the same connected component of $\mathcal{S}$, one can define $y_{e}(\tau, \cdot)$, with $\tau \in[0,1]$, a $\mathcal{C}^{2}$ path in $\mathcal{S}$ connecting $y_{e, 1}$ to $y_{e, 2}$, as well as the associated path of boundary inputs $u_{e}(\tau)=\frac{\partial y_{e}}{\partial x}(\tau, L)$. Now, a possible attempt would be to extend the approach of [16] by defining the path of system outputs $z_{e}(\tau)=\frac{\partial y_{e}}{\partial x}(\tau, 0)$ associated with $y_{e}(\tau, \cdot)$. In this setting, the objective would be to study the deviations of the system output $z(t)=\frac{\partial y}{\partial x}(t, 0)$ with respect to the quasistatic path $y_{e}(\epsilon t, \cdot)$ by introducing $z_{\delta}(t, x)=z(t)-z_{e}(\epsilon t)$. In this configuration, $r(t)=z_{e}(\epsilon t)$ plays the role of a slowly time-varying reference input. 
It was shown in [16] that such a path can be used in order to steer the system (1) from the steady-state $y_{e, 1}$ to the steady-state $y_{e, 2}$ by means of a boundary control input $u$ taking advantage of quasi-static deformations. Specifically, for $\epsilon>0$ small enough, the authors studied the deviations of the system trajectory with respect to the quasi-static path $y_{e}(\epsilon t, \cdot)$ by introducing:

$$
\begin{aligned}
y_{\delta}(t, x) & =y(t, x)-y_{e}(\epsilon t, x), \\
u_{\delta}(t) & =u(t)-u_{e}(\epsilon t)
\end{aligned}
$$

for $t \in[0,1 / \epsilon]$ and $x \in[0, L]$. The preliminary feedback still takes the form (6). Due to the quasi-static deformations-based approach and using a Taylor expansion as in (3a), the design of the auxiliary control input $v(t)$ requires the introducing of the following family of wave operators parametrized by $\tau \in[0,1]$ :

$$
\mathcal{A}(\tau)=\left(\begin{array}{cc}
0 & \mathrm{Id} \\
\mathcal{A}_{0}(\tau) & 0
\end{array}\right)
$$

with $\mathcal{A}_{0}(\tau)=\Delta+f^{\prime}\left(y_{e}(\tau, \cdot)\right)$ Id defined on the domain

$$
\begin{aligned}
D(\mathcal{A}(\tau))=\left\{\left(w^{1}, w^{2}\right) \in \mathcal{H}:\right. & w^{1} \in H^{2}(0, L), w^{2} \in H^{1}(0, L), \\
& \left.w^{2}(0)=0,\left(w^{1}\right)^{\prime}(L)+\alpha w^{2}(L)=0\right\} .
\end{aligned}
$$

Following [16, Lem. 2], this family of operators admits a family $\left(e_{k}(\tau, \cdot)\right)_{k \in \mathbb{Z}}$ of Riesz-bases formed by generalized eigenvectors of $\mathcal{A}(\tau)$, associated to the eigenvalues $\left(\lambda_{k}(\tau)\right)_{k \in \mathbb{Z}}$ and with dual Riesz basis $\left(f_{k}(\tau, \cdot)\right)_{k \in \mathbb{Z}}$, with properties similar to the ones of (2) but with an integer $n_{0} \geqslant 0$ that is uniform with respect to $\tau \in[0,1]$. Without loss of generality, this latter integer can be selected such that $|k| \geqslant n_{0}+1$ implies Re $\lambda_{k}<-1$. Moreover, the aforementioned family of Riesz-bases is uniform with respect to $\tau \in[0,1]$ in the sense that the constants $m_{R}, M_{R}$ of Definition 2 can be selected independently of $\tau \in[0,1]$. These key properties allowed the authors of [16] to design a control law of the form $v(t)=K(\epsilon t) X(t)$. The matrix $K(\tau)$, parametrized by $\tau \in[0,1]$, is obtained based on an augmented finite-dimensional LTI system, also parametrized by $\tau \in[0,1]$, which in particular captures the first modes of $\mathcal{A}(\tau)$ characterized by the integers $-n_{0} \leqslant k \leqslant n_{0}$. The vector $X(t)$ captures, in addition to a number of integral components, the projection of the system trajectory $W(t, \cdot)$ onto the vector space spanned by $\left(e_{k}(t \epsilon, \cdot)\right)_{|k| \leqslant n_{0}}$. The stability property of the resulting closed-loop system was assessed through the study of a suitable Lyapunov functional, yielding the following result [16, Thm. 1]. For the wave equation (1) with initial condition set as the steady-state $y_{e, 1}$ and for the control input selected as above: for every $\delta>0$, there exists $\epsilon_{1}>0$ so that, for every $\epsilon \in\left(0, \epsilon_{1}\right]$, we have

$$
\left\|\frac{\partial y}{\partial x}(1 / \epsilon, \cdot)-\frac{\mathrm{d} y_{e, 2}}{\mathrm{~d} x}\right\|_{L^{2}(0, L)}+\left\|\frac{\partial y}{\partial t}(1 / \epsilon, \cdot)\right\|_{L^{2}(0, L)} \leqslant \delta .
$$

In conclusion, it is of interest to evaluate the possible extension of our PI regulation procedure to the case of quasi-steady deformations as described above.

\subsection{System of one-dimensional partial differential equations}

In Section 6.2, we have mentioned as a completely open issue the general multi-dimensional case, which is very challenging. As an intermediate case between 1-D and multi-D, we may consider the case of systems of one-dimensional partial differential equations. A line of research that is of great interest is to consider coupled scalar one-dimensional PDEs, for instance of the form

$$
\frac{\partial^{2} y}{\partial t^{2}}=a \frac{\partial^{2} y}{\partial x^{2}}+c y+d z
$$




$$
\frac{\partial^{2} z}{\partial t^{2}}=b \frac{\partial^{2} y}{\partial x^{2}}+e y+f z
$$

which are 1-D coupled wave-like equations, with various possible controls and with various outputs (e.g., Neumann boundary control and Neumann regulated output like in this article).

Of great interest too, would be to replace the above wave equation in $z$, with a heat-like equation in $z$, that is, consider a 1-D wave-like equation that is coupled with a 1-D heat-like equation in $z$. In that case, we expect new phenomena, emerging from the interesting coupling between a parabolic and a hyperbolic equation. Actually, even the case of coupled 1-D heat-like equations does not seem to have been considered in the literature concerning PI issues.

It would be very interesting to address these problems. They indeed have attracted much attention for controllability issues and many powerful techniques have been introduced to treat such coupled systems (see, e.g., [1, 2, 3, 4, 5, 6, 31]). This open issue is a future line of research.

\section{A Annex - Proof of Lemma 2}

From the definition of the operator $\mathcal{A}$ given by (13), $\lambda \in \mathbb{C}$ is an eigenvalue of $\mathcal{A}$ associated with the nonzero eigenvector $w=\left(w^{1}, w^{2}\right) \in D(\mathcal{A})$ if and only if $w^{2}=\lambda w^{1}$ and

$$
\begin{aligned}
& \left(w^{1}\right)^{\prime \prime}+f^{\prime}\left(y_{e}\right) w^{1}=\lambda^{2} w^{1}, \\
& w^{1}(0)=0, \quad\left(w^{1}\right)^{\prime}(L)+\alpha \lambda w^{1}(L)=0
\end{aligned}
$$

for $x \in(0, L)$. Then, for $|\lambda| \rightarrow+\infty$, we obtain for $x \in[0, L]$ that

$$
w^{1}(x)=\sinh \left(\sqrt{\lambda^{2}+O(1)} x\right), \quad\left(w^{1}\right)^{\prime}(x)=\sqrt{\lambda^{2}+O(1)} \cosh \left(\sqrt{\lambda^{2}+O(1)} x\right),
$$

uniformly with respect to $x \in[0, L]$. Using now the right boundary conditions, we obtain the existence of an integer $k \in \mathbb{Z}$ such that, as $|k| \rightarrow+\infty$,

$$
\lambda_{k}=\frac{1}{2 L} \log \left(\frac{\alpha-1}{\alpha+1}\right)+i \frac{k \pi}{L}+O\left(\frac{1}{|k|}\right) .
$$

Then, an associated unit eigenvector is given by

$$
e_{k}=\frac{1}{A_{k}}\left(\sinh \left(\sqrt{\lambda_{k}^{2}+O(1)} x\right), \lambda_{k} \sinh \left(\sqrt{\lambda_{k}^{2}+O(1) x}\right)\right)
$$

where, recalling that $\alpha>1$ and introducing $\beta=-\frac{1}{2 L} \log \left(\frac{\alpha-1}{\alpha+1}\right)>0$,

$$
A_{k}=\left|\lambda_{k}\right| \sqrt{\frac{\sinh (2 \beta L)}{2 \beta}+O\left(\frac{1}{|k|}\right)} .
$$

In particular, $\left(e_{k}^{1}\right)^{\prime}(0)=O(1)$ as $|k| \rightarrow+\infty$, showing item 7 .

We show that the eigenvalues of $\mathcal{A}$ are geometrically simple (item 2). To do so, assume that $w_{i}=\left(w_{i}^{1}, w_{i}^{2}\right) \in D(\mathcal{A})$, with $i \in\{1,2\}$, are two eigenvectors of $\mathcal{A}$ associated with the same eigenvalue $\lambda \in \mathbb{C}$. We note that $w_{i}(L) \neq 0$ because otherwise $\left(w_{i}^{1}\right)^{\prime}(L)=-\alpha \lambda w_{i}^{1}(l)=0$ hence, by cauchy uniqueness, $w_{i}^{1}=0$ and thus $w_{i}^{2}=\lambda w_{i}^{1}=0$, giving the contradiction $w=0$. Then the function $g$ defined by $g=w_{2}^{1}(L) w_{1}^{1}-w_{1}^{1}(L) w_{2}^{1} \neq 0$ satisfies

$$
g^{\prime \prime}+f^{\prime}\left(y_{e}\right) g=\lambda^{2} g,
$$




$$
g(L)=g^{\prime}(L)=0
$$

implying $g=0$. Recalling that $w_{i}^{2}=\lambda w_{i}^{1}$, this shows that $w_{1}$ and $w_{2}$ are not linearly independent.

Recalling that $\mathcal{A}$ has compact resolvent, we denote by $\left(e_{k}\right)_{k \in \mathbb{Z}}$ a complete set of unit generalized eigenfunctions of $\mathcal{A}$ associated with the eigenvalues $\left(\lambda_{k}\right)_{k \in \mathbb{Z}}$ [21]. We are going to apply Bari's theorem [21] to show that $\left(e_{k}\right)_{k \in \mathbb{Z}}$ is a Riesz basis. To do so, we need a Riesz basis of reference. Based on the definition the operator $\mathcal{A}$ given by (13), we consider the below operator, obtained by removing the contribution of the terme $f^{\prime}\left(y_{e}\right) \mathrm{Id}$,

$$
\mathcal{A}_{t r}=\left(\begin{array}{cc}
0 & \mathrm{Id} \\
\Delta & 0
\end{array}\right)
$$

defined on the same domain as $\mathcal{A}$. We know from [38, Section 4] that $\mathcal{A}_{t r}$ admits a Riesz basis of eigenvectors $\left(\phi_{k}\right)_{k \in \mathbb{Z}}$ associated with the eigenvalues $\left(\mu_{k}\right)_{k \in \mathbb{Z}}$ given for any $k \in \mathbb{Z}$ by

$$
\mu_{k}=\frac{1}{2 L} \log \left(\frac{\alpha-1}{\alpha+1}\right)+i \frac{k \pi}{L}
$$

and

$$
\phi_{k}=\frac{1}{B_{k}}\left(\sinh \left(\mu_{k} x\right), \mu_{k} \sinh \left(\mu_{k} x\right)\right)
$$

where, recalling that $\beta=-\frac{1}{2 L} \log \left(\frac{\alpha-1}{\alpha+1}\right)>0$,

$$
B_{k}=\frac{1}{L \sqrt{2 \beta}} \sqrt{\left(\beta^{2} L^{2}+k^{2} \pi^{2}\right) \sinh (2 \beta L)} .
$$

We deduce that

$$
e_{k}=\phi_{k}+O\left(\frac{1}{|k|}\right)
$$

in $\mathcal{H}$-norm as $|k| \rightarrow+\infty$. Hence $\left(e_{k}\right)_{k \in \mathbb{Z}}$ is quadratically close to the Riesz basis $\left(\phi_{k}\right)_{k \in \mathbb{Z}}$. Then, with the results of [22, Lemma 6.2 and Theorem 6.3] relying on Bari's theorem, we obtain that $\left(e_{k}\right)_{k \in \mathbb{Z}}$ is a Riesz basis. Introducing $\left(f_{k}\right)_{k \in \mathbb{Z}}$ as the Dual Riesz basis of $\left(e_{k}\right)_{k \in \mathbb{Z}}$, items $1,3,4$, and 6 hold true. Finally, an homotopy argument using the operator $\mathcal{A}_{t r}$ shows that the algebraic multiplicity of the real eigenvalues of $\mathcal{A}$ is odd, yielding item 5 .

\section{References}

[1] F. Alabau-Boussouira, "Indirect boundary stabilization of weakly coupled hyperbolic systems," SIAM J. Control Optim., vol. 41, no. 2, pp. 511-541, 2002.

[2] — "On the influence of the coupling on the dynamics of single-observed cascade systems of PDE's," Math. Control Relat. Fields, vol. 5, no. 1, pp. 1-30, 2015.

[3] F. Alabau-Boussouira and M. Léautaud, "Indirect controllability of locally coupled wave-type systems and applications," J. Math. Pures Appl. (9), vol. 99, no. 5, pp. 544-576, 2013.

[4] F. Ammar-Khodja, A. Benabdallah, M. González-Burgos, and L. de Teresa, "The Kalman condition for the boundary controllability of coupled parabolic systems. Bounds on biorthogonal families to complex matrix exponentials," J. Math. Pures Appl. (9), vol. 96, no. 6, pp. $555-590,2011$. 
[5] — - "Recent results on the controllability of linear coupled parabolic problems: a survey," Math. Control Relat. Fields, vol. 1, no. 3, pp. 267-306, 2011.

[6] _ - "Minimal time for the null controllability of parabolic systems: the effect of the condensation index of complex sequences," J. Funct. Anal., vol. 267, no. 7, pp. 2077-2151, 2014.

[7] Z. Artstein, "Linear systems with delayed controls: a reduction," IEEE Transactions on Automatic Control, vol. 27, no. 4, pp. 869-879, 1982.

[8] K. J. Åström and T. Hägglund, PID controllers: theory, design, and tuning. Instrument society of America Research Triangle Park, NC, 1995, vol. 2.

[9] K. J. Astrom and R. M. Murray, "Feedback systems," in An introduction for Scientists and Engineers. Princeton Unvirsity Press, 2008.

[10] M. Barreau, F. Gouaisbaut, and A. Seuret, "Practical stabilization of a drilling pipe under friction with a PI-controller," arXiv preprint arXiv:1904.10658, 2019.

[11] G. Bastin and J.-M. Coron, "Exponential stability of PI control for Saint-Venant equations with a friction term," Methods and Applications of Analysis, vol. 26, no. 2, pp. 101-112, 2019.

[12] G. Bastin, J.-M. Coron, and S. O. Tamasoiu, "Stability of linear density-flow hyperbolic systems under PI boundary control," Automatica, vol. 53, pp. 37-42, 2015.

[13] D. Bresch-Pietri, C. Prieur, and E. Trélat, "New formulation of predictors for finitedimensional linear control systems with input delay," Systems 83 Control Letters, vol. 113, pp. 9-16, 2018.

[14] J.-M. Coron and A. Hayat, "PI controllers for 1-D nonlinear transport equation," IEEE Transactions on Automatic Control, vol. 64, no. 11, pp. 4570-4582, 2019.

[15] J.-M. Coron and E. Trélat, "Global steady-state controllability of one-dimensional semilinear heat equations," SIAM Journal on Control and Optimization, vol. 43, no. 2, pp. 549-569, 2004 .

[16] — - "Global steady-state stabilization and controllability of 1D semilinear wave equations," Communications in Contemporary Mathematics, vol. 8, no. 04, pp. 535-567, 2006.

[17] R. Datko, "Two examples of ill-posedness with respect to time delays revisited," IEEE transactions on Automatic Control, vol. 42, no. 4, pp. 511-515, 1997.

[18] I. A. Djebour, T. Takahashi, and J. Valein, "Feedback stabilization of parabolic systems with input delay," 2020.

[19] V. Dos Santos, G. Bastin, J.-M. Coron, and B. d'Andréa Novel, "Boundary control with integral action for hyperbolic systems of conservation laws: Stability and experiments," $A u$ tomatica, vol. 44, no. 5, pp. 1310-1318, 2008.

[20] V. Dos Santos Martins, Y. Wu, and M. Rodrigues, "Design of a proportional integral control using operator theory for infinite dimensional hyperbolic systems," IEEE Transactions on Control Systems Technology, vol. 22, no. 5, pp. 2024-2030, 2014.

[21] I. Gohberg and M. G. Krein, Introduction to the theory of linear nonselfadjoint operators. American Mathematical Soc., 1978, vol. 18. 
[22] B.-Z. Guo, "Riesz basis approach to the stabilization of a flexible beam with a tip mass," SIAM Journal on Control and Optimization, vol. 39, no. 6, pp. 1736-1747, 2001.

[23] A. Hayat, "PI controller for the general Saint-Venant equations," HAL preprint hal$01827988 v 3 f, 2019$.

[24] H. K. Khalil, Nonlinear systems. Macmillan Publishing Company, New York, 1992.

[25] V. Kolmanovskii and A. Myshkis, Applied theory of functional differential equations. Springer Science \& Business Media, 2012, vol. 85.

[26] M. Krstic and A. Smyshlyaev, Boundary control of PDEs, ser. Advances in Design and Control. Society for Industrial and Applied Mathematics (SIAM), Philadelphia, PA, 2008, vol. 16, a course on backstepping designs.

[27] P.-O. Lamare and N. Bekiaris-Liberis, "Control of $2 \times 2$ linear hyperbolic systems: Backstepping-based trajectory generation and PI-based tracking," Systems $\&$ Control Letters, vol. 86, pp. 24-33, 2015.

[28] H. Lhachemi and C. Prieur, "Feedback stabilization of a class of diagonal infinite-dimensional systems with delay boundary control," IEEE Transactions on Automatic Control, 2021, in press.

[29] H. Lhachemi, C. Prieur, and E. Trélat, "PI regulation of a reaction-diffusion equation with delayed boundary control," IEEE Transactions on Automatic Control, 2021, in press.

[30] H. Lhachemi and R. Shorten, "Boundary feedback stabilization of a reaction-diffusion equation with robin boundary conditions and state-delay," Automatica, vol. 116, p. 108931, 2020.

[31] T. Liard and P. Lissy, "A Kalman rank condition for the indirect controllability of coupled systems of linear operator groups," Math. Control Signals Systems, vol. 29, no. 2, pp. Art. 9, $35,2017$.

[32] H. Logemann and H. Zwart, "On robust PI-control of infinite-dimensional systems," SIAM journal on control and optimization, vol. 30, no. 3, pp. 573-593, 1992.

[33] A. Mironchenko and C. Prieur, "Input-to-state stability of infinite-dimensional systems: recent results and open questions," arXiv preprint arXiv:1910.01714, 2019.

[34] A. Pazy, Semigroups of linear operators and applications to partial differential equations. Springer Science \& Business Media, 2012, vol. 44.

[35] S. Pohjolainen, "Robust multivariable PI-controller for infinite dimensional systems," IEEE Transactions on Automatic Control, vol. 27, no. 1, pp. 17-30, 1982.

[36] _ - "Robust controller for systems with exponentially stable strongly continuous semigroups," Journal of mathematical analysis and applications, vol. 111, no. 2, pp. 622-636, 1985.

[37] M. Rodrigues, Y. Wu, S. Aberkane, and V. D. S. Martins, "LMI \& BMI technics for the design of a PI control for irrigation channels," in 2013 European Control Conference (ECC). IEEE, 2013, pp. 3895-3900.

[38] D. L. Russell, "Controllability and stabilizability theory for linear partial differential equations: recent progress and open questions," SIAM Review, vol. 20, no. 4, pp. 639-739, 1978. 
[39] M. Schmidt and E. Trélat, "Controllability of Couette flows," Commun. Pure Appl. Anal., vol. 5, no. 1, pp. 201-211, 2006.

[40] E. D. Sontag, Mathematical control theory, ser. Texts in Applied Mathematics. SpringerVerlag, New York, 1990, vol. 6, deterministic finite-dimensional systems.

[41] A. Terrand-Jeanne, V. Andrieu, V. D. S. Martins, and C.-Z. Xu, "Lyapunov functionals for output regulation of exponentially stable semigroups via integral action and application to hyperbolic systems," in 2018 IEEE Conference on Decision and Control (CDC), Miami Beach, FL, USA, 2018, pp. 4631-4636.

[42] — - "Adding integral action for open-loop exponentially stable semigroups and application to boundary control of PDE systems," arXiv preprint arXiv:1901.02208, 2019.

[43] A. Terrand-Jeanne, V. Andrieu, M. Tayakout-Fayolle, and V. D. S. Martins, "Regulation of inhomogeneous drilling model with a PI controller," IEEE Transactions on Automatic Control, vol. 65 , no. 1 , pp. 58-71, 2020.

[44] E. Trélat, Contrôle optimal, ser. Mathématiques Concrètes. [Concrete Mathematics]. Vuibert, Paris, 2005, théorie \& applications. [Theory and applications].

[45] N.-T. Trinh, V. Andrieu, and C.-Z. Xu, "Design of integral controllers for nonlinear systems governed by scalar hyperbolic partial differential equations," IEEE Transactions on Automatic Control, vol. 62, no. 9, pp. 4527-4536, 2017.

[46] M. Tucsnak and G. Weiss, Observation and control for operator semigroups. Springer Science \& Business Media, 2009.

[47] C.-Z. Xu and H. Jerbi, "A robust PI-controller for infinite-dimensional systems," International Journal of Control, vol. 61, no. 1, pp. 33-45, 1995.

[48] C.-Z. Xu and G. Sallet, "Multivariable boundary PI control and regulation of a fluid flow system," Mathematical Control and Related Fields, vol. 4, no. 4, pp. 501-520, 2014.

[49] J. Zabczyk, Mathematical control theory: an introduction, ser. Systems \& Control: Foundations \& Applications. Birkhäuser Boston, Inc., Boston, MA, 1992. 